\title{
Approximations by multivariate sublinear and Max-product operators under convexity
}

https://doi.org/10.1515/dema-2018-0008

Received November 6, 2017; accepted April 6, 2018

Abstract: Here we search quantitatively under convexity the approximation of multivariate function by general multivariate positive sublinear operators with applications to multivariate Max-product operators. These are of Bernstein type, of Favard-Szász-Mirakjan type, of Baskakov type, of sampling type, of Lagrange interpolation type and of Hermite-Fejér interpolation type. Our results are both: under the presence of smoothness and without any smoothness assumption on the function to be approximated which fulfills a convexity assumption.

Keywords: multivariate positive sublinear operators, multivariate Max-product operators, multivariate modulus of continuity, convexity

MSC: 41A17, 41A25, 41A36, 41A63

\section{Background}

In this article we study under convexity quantitatively the approximation properties of multivariate Maxproduct operators to the unit. These are special cases of multivariate positive sublinear operators. We also give general results regarding the convergence to the unit of multivariate positive sublinear operators under convexity. Special emphasis is given to our study about approximation also under the presence of smoothness. Our work is inspired from [1].

Let $Q$ be a compact and convex subset of $\mathbb{R}^{k}, k \in \mathbb{N}-\{1\}$, and let $x_{0}:=\left(x_{01}, \ldots, x_{0 k}\right) \in Q^{o}$ be fixed. Let $f \in C^{n}(Q)$ and suppose that each $n$th partial derivative $f_{\alpha}=\frac{\partial^{\alpha} f}{\partial x^{\alpha}}$, where $\alpha:=\left(\alpha_{1}, \ldots, \alpha_{k}\right), \alpha_{i} \in \mathbb{Z}^{+}, i=1, \ldots, k$, and $|\alpha|:=\sum_{i=1}^{k} \alpha_{i}=n$, has relative to $Q$ and the $l_{1}$-norm $\|\cdot\|$, a modulus of continuity $\omega_{1}\left(f_{\alpha}, h\right) \leq w$, where $h$ and $w$ are fixed positive numbers. Here

$$
\omega_{1}\left(f_{\alpha}, h\right):=\sup _{\substack{x, y \in Q \\\|x-y\|_{l_{1}} \leq h}}\left|f_{\alpha}(x)-f_{\alpha}(y)\right| .
$$

We assume that the ball $B\left(x_{0}, h\right) \subset Q$. We also assume that $f_{\alpha}\left(x_{0}\right)=0$, for all $\alpha:|\alpha|=1, \ldots, n$, and $\left|f_{\alpha}(x)\right|$ is convex in $x$, for all $\alpha:|\alpha|=n$.

The $j$ th derivative of $g_{z}(t)=f\left(x_{0}+t\left(z-x_{0}\right)\right),\left(z=\left(z_{1}, \ldots, z_{k}\right) \in Q\right)$ is given by

$$
g_{z}^{(j)}(t)=\left[\left(\sum_{i=1}^{k}\left(z_{i}-x_{0 i}\right) \frac{\partial}{\partial x_{i}}\right)^{j} f\right]\left(x_{01}+t\left(z_{1}-x_{01}\right), \ldots, x_{0 k}+t\left(z_{k}-x_{0 k}\right)\right) .
$$

Consequently, it holds that

$$
f\left(z_{1}, \ldots, z_{k}\right)=g_{z}(1)=\sum_{j=0}^{n} \frac{g_{z}^{(j)}(0)}{j !}+R_{n}(z, 0)
$$

\footnotetext{
^Corresponding Author: George A. Anastassiou: Department of Mathematical Sciences, University of Memphis, Memphis,
} TN 38152, U.S.A., E-mail: ganastss@memphis.edu 
where

$$
R_{n}(z, 0):=\int_{0}^{1}\left(\int_{0}^{t_{1}} \ldots\left(\int_{0}^{t_{n-1}}\left(g_{z}^{(n)}\left(t_{n}\right)-g_{z}^{(n)}(0)\right) d t_{n}\right) \ldots\right) d t_{1} .
$$

We apply Lemma 8.1.1, [2, p. 243], to $\left(f_{\alpha}\left(x_{0}+t\left(z-x_{0}\right)\right)-f_{\alpha}\left(x_{0}\right)\right)$ as a function of $z$, when $\omega_{1}\left(f_{\alpha}, h\right) \leq w$.

$$
\left|f_{\alpha}\left(x_{0}+t\left(z-x_{0}\right)\right)-f_{\alpha}\left(x_{0}\right)\right| \leq w \frac{t\left\|z-x_{0}\right\|}{h},
$$

for all $t \geq 0$.

Let $Q$ be a compact and convex subset of $\mathbb{R}^{k}, k \in \mathbb{N}-\{1\}, x_{0} \in Q$ fixed, $f \in C^{n}(Q)$. Then for $j=1, \ldots, n$, we have

$$
g_{z}^{(j)}(0)=\sum_{\substack{\alpha:=\left(\alpha_{1}, \ldots, \alpha_{k}\right), \alpha_{i} \in \mathbb{Z}^{+}, i=1, \ldots, k,|\alpha|:=\sum_{i=1}^{k} \alpha_{i}=j}}\left(\frac{j !}{\prod_{i=1}^{k} \alpha_{i} !}\right)\left(\prod_{i=1}^{k}\left(z_{i}-x_{0 i}\right)^{\alpha_{i}}\right) f_{\alpha}\left(x_{0}\right) .
$$

If $f_{\alpha}\left(x_{0}\right)=0$, for all $\alpha:|\alpha|=1, \ldots, n$, then $g_{z}^{(j)}(0)=0, j=1, \ldots, n$, and by (3) we get

$$
f(z)-f\left(x_{0}\right)=R_{n}(z, 0) \text {. }
$$

It follows from (2) that

$$
\begin{gathered}
\left|R_{n}(z, 0)\right| \leq \\
\int_{0}^{1}\left[\int_{0}^{t_{1}} \ldots\left[\int_{0}^{t_{n-1}}\left(\sum_{|\alpha|=n} \frac{n ! \prod_{i=1}^{k}\left|z_{i}-x_{0 i}\right|^{\alpha_{i}}}{\alpha_{1} ! \ldots \alpha_{k} !} \frac{w}{h}\left\|z-x_{0}\right\| t_{n}\right) d t_{n}\right] \ldots\right] d t_{1} \\
=\frac{w}{h} \frac{\left\|z-x_{0}\right\|^{n+1}}{(n+1) !} .
\end{gathered}
$$

Therefore, it holds that

$$
\left|R_{n}(z, 0)\right| \leq \frac{w}{h} \frac{\left\|z-x_{0}\right\|^{n+1}}{(n+1) !} \text {, for all } z \in Q
$$

Note that $g_{z}(0)=f\left(z_{0}\right)$.

That is

$$
\left|f(z)-f\left(x_{0}\right)\right| \leq \frac{w}{h} \frac{\left\|z-x_{0}\right\|^{n+1}}{(n+1) !}, \forall z \in Q .
$$

We have proved the following fundamental result:

Theorem 1. Let $Q$ with the $l_{1}$-norm $\|\cdot\|$, be a compact and convex subset of $\mathbb{R}^{k}, k \geq 1$, let $x_{0}=\left(x_{01}, \ldots, x_{0 k}\right) \in$ $Q^{o}$ be fixed. Let $f \in C^{n}(Q), n \in \mathbb{N}$ and suppose that each nth partial derivative $f_{\alpha}=\frac{\partial^{\alpha} f}{\partial x^{\alpha}}$, where $\alpha=\left(\alpha_{1}, \ldots, \alpha_{k}\right)$, $\alpha_{i} \geq 0, i=1, \ldots, k$, and $|\alpha|=\sum_{i=1}^{k} \alpha_{i}=n$ has, relative to $Q$ and the $l_{1}$-norm, a modulus of continuity $\omega_{1}\left(f_{\alpha}, h\right)$, and that each $\left|f_{\alpha}(x)\right|$ is a convex function of $x \in Q$, all $\alpha:|\alpha|=n$. Assume further that $f_{\alpha}\left(x_{0}\right)=0$, for all $\alpha:|\alpha|=1, \ldots, n$; and $h>0$ such that the ball in $\mathbb{R}^{k}: B\left(x_{0}, h\right)$ is contained in $Q$. Then

$$
\left\|f(z)-f\left(x_{0}\right)\right\| \leq \frac{\left(\max _{\alpha:|\alpha|=n} \omega_{1}\left(f_{\alpha}, h\right)\right)}{h} \frac{\left\|z-x_{0}\right\|^{n+1}}{(n+1) !}, \forall z \in Q .
$$

In conclusion we have

Theorem 2. Let $Q$ with the $l_{1}$-norm $\|\cdot\|$, be a compact and convex subset of $\mathbb{R}^{k}, k \geq 1$, let $x=\left(x_{1}, \ldots, x_{k}\right) \in Q^{o}$ be fixed. Let $f \in C^{n}(Q), n \in \mathbb{N}$ and suppose that each nth partial derivative $f_{\alpha}=\frac{\partial^{\alpha} f}{\partial x^{\alpha}}$, where $\alpha=\left(\alpha_{1}, \ldots, \alpha_{k}\right)$, $\alpha_{i} \geq 0, i=1, \ldots, k$, and $|\alpha|=\sum_{i=1}^{k} \alpha_{i}=n$, has relative to $Q$ and the $l_{1}$-norm a modulus of continuity $\omega_{1}\left(f_{\alpha}, h\right)$, 
and that each $\left|f_{\alpha}(t)\right|$ is a convex function of $t \in Q$, for all $\alpha:|\alpha|=n$. Assume further that $f_{\alpha}(x)=0$, for all $\alpha:|\alpha|=1, \ldots, n$, and $h>0$ such that the ball in $\mathbb{R}^{k}: B(x, h) \subset Q$. Then

$$
\begin{gathered}
\|f(t)-f(x)\| \leq \frac{\left(\max _{\alpha:|\alpha|=n} \omega_{1}\left(f_{\alpha}, h\right)\right)}{h} \frac{\|t-x\|^{n+1}}{(n+1) !} \leq \\
\frac{\left(\max _{\alpha:|\alpha|=n} \omega_{1}\left(f_{\alpha}, h\right)\right)}{h(n+1) !} k^{n}\left(\sum_{i=1}^{k}\left|t_{i}-x_{i}\right|^{n+1}\right),
\end{gathered}
$$

for all $t \in Q$, where $t=\left(t_{1}, \ldots, t_{k}\right)$.

Proof. By Theorem 1 and a convexity argument.

We need

Definition 3. Let $Q$ be a compact and convex subset of $\mathbb{R}^{k}, k \in \mathbb{N}-\{1\}$. Here we denote

$$
C_{+}(Q)=\left\{f: Q \rightarrow \mathbb{R}_{+} \text {and continuous }\right\}
$$

Let $L_{N}: C_{+}(Q) \rightarrow C_{+}(Q), N \in \mathbb{N}$, be a sequence of operators satisfying the following properties:

(i) (positive homogeneous)

$$
L_{N}(\alpha f)=\alpha L_{N}(f), \forall \alpha \geq 0, f \in C_{+}(Q) ;
$$

(ii) (monotonicity)

if $f, g \in C_{+}(Q)$ satisfy $f \leq g$, then

$$
L_{N}(f) \leq L_{N}(g), \forall N \in \mathbb{N},
$$

and

(iii) (subadditivity)

$$
L_{N}(f+g) \leq L_{N}(f)+L_{N}(g), \forall f, g \in C_{+}(Q) .
$$

We call $L_{N}$ positive sublinear operators.

Remark 4. (to Definition 3) Let $f, g \in C_{+}(Q)$. We see that $f=f-g+g \leq|f-g|+g$. Then $L_{N}(f) \leq L_{N}(|f-g|)+$ $L_{N}(g)$, and $L_{N}(f)-L_{N}(g) \leq L_{N}(|f-g|)$.

Similarly, $g=g-f+f \leq|g-f|+f$, hence $L_{N}(g) \leq L_{N}(|f-g|)+L_{N}(f)$, and $L_{N}(g)-L_{N}(f) \leq L_{N}(|f-g|)$.

Consequently, it holds that

$$
\left|L_{N}(f)(x)-L_{N}(g)(x)\right| \leq L_{N}(|f-g|)(x), \forall x \in Q .
$$

In this article we treat $L_{N}: L_{N}(1)=1$.

We observe that

$$
\begin{gathered}
\left|L_{N}(f)(x)-f(x)\right|=\left|L_{N}(f)(x)-L_{N}(f(x))(x)\right| \stackrel{(17)}{\leq} \\
L_{N}(|f(\cdot)-f(x)|)(x), \forall x \in Q .
\end{gathered}
$$

We give

Theorem 5. All as in Theorem 2, $f \in C^{n}\left(Q, \mathbb{R}_{+}\right)$. Let $\left\{L_{N}\right\}_{N \in \mathbb{N}}$ be positive sublinear operators mapping $C_{+}(Q)$ into itself, such that $L_{N}(1)=1$. Then

$$
\left|L_{N}(f)(x)-f(x)\right| \leq \frac{\left(\max _{\alpha:|\alpha|=n} \omega_{1}\left(f_{\alpha}, h\right)\right) k^{n}}{h(n+1) !}\left(\sum_{i=1}^{k} L_{N}\left(\left|t_{i}-x_{i}\right|^{n+1}\right)(x)\right),
$$

for all $N \in \mathbb{N}$. 
Proof. By Theorem 2, see Definition 3, and by (18).

We need

The Maximum Multiplicative Principle 6. Here $\vee$ stands for maximum. Let $\alpha_{i}>0, i=1, \ldots, n ; \beta_{j}>0$, $j=1, \ldots, m$. Then

$$
\vee_{i=1}^{n} \vee_{j=1}^{m} \alpha_{i} \beta_{j}=\left(\vee_{i=1}^{n} \alpha_{i}\right)\left(\vee_{j=1}^{m} \beta_{j}\right)
$$

Proof. Obvious.

We make

Remark 7. In $[1, p .10]$, the authors introduced the basic Max-product Bernstein operators

$$
B_{N}^{(M)}(f)(x)=\frac{\bigvee_{k=0}^{N} p_{N, k}(x) f\left(\frac{k}{N}\right)}{\bigvee_{k=0}^{N} p_{N, k}(x)}, N \in \mathbb{N},
$$

where $p_{N, k}(x)=\left(\begin{array}{c}N \\ k\end{array}\right) x^{k}(1-x)^{N-k}, x \in[0,1]$, and $f:[0,1] \rightarrow \mathbb{R}_{+}$is continuous.

In $[1, p .31]$, they proved that

$$
B_{N}^{(M)}(|\cdot-x|)(x) \leq \frac{6}{\sqrt{N+1}}, \forall x \in[0,1], \forall N \in \mathbb{N} .
$$

And in [3] was proved that

$$
B_{N}^{(M)}\left(|\cdot-x|^{m}\right)(x) \leq \frac{6}{\sqrt{N+1}}, \forall x \in[0,1], \forall m, N \in \mathbb{N} .
$$

Under our convexity conditions the derived convergence inequalities are very elegant and compact.

\section{Main Results}

From now on $Q=[0,1]^{k}, k \in \mathbb{N}-\{1\}$, except otherwise specified.

We mention

Definition 8. Let $f \in C_{+}\left([0,1]^{k}\right)$, and $\vec{N}=\left(N_{1}, \ldots, N_{k}\right) \in \mathbb{N}^{k}$. We define the multivariate Max-product Bernstein operators as follows:

$$
\begin{gathered}
B_{\vec{N}}^{(M)}(f)(x):= \\
\frac{\vee_{i_{1}=0}^{N_{1}} \vee_{i_{2}=0}^{N_{2}} \ldots \vee_{i_{k}=0}^{N_{k}} p_{N_{1}, i_{1}}\left(x_{1}\right) p_{N_{2}, i_{2}}\left(x_{2}\right) \ldots p_{N_{k}, i_{k}}\left(x_{k}\right) f\left(\frac{i_{1}}{N_{1}}, \ldots, \frac{i_{k}}{N_{k}}\right)}{\vee_{i_{1}=0}^{N_{1}} \vee_{i_{2}=0}^{N_{2}} \ldots \vee_{i_{k}=0}^{N_{k}} p_{N_{1}, i_{1}}\left(x_{1}\right) p_{N_{2}, i_{2}}\left(x_{2}\right) \ldots p_{N_{k}, i_{k}}\left(x_{k}\right)},
\end{gathered}
$$

for all $x=\left(x_{1}, \ldots, x_{k}\right) \in[0,1]^{k}$. Call $N_{\min }:=\min \left\{N_{1}, \ldots, N_{k}\right\}$.

The operators $B_{\vec{N}}^{(M)}(f)(x)$ are positive sublinear and they map $C_{+}\left([0,1]^{k}\right)$ into itself, and $B_{\vec{N}}^{(M)}(1)=1$.

See also [1, p. 123] for the bivariate case. We also have

$$
\begin{gathered}
B_{\vec{N}}^{(M)}(f)(x):= \\
\frac{\vee_{i_{1}=0}^{N_{1}} \vee_{i_{2}=0}^{N_{2}} \ldots \vee_{i_{k}=0}^{N_{k}} p_{N_{1}, i_{1}}\left(x_{1}\right) p_{N_{2}, i_{2}}\left(x_{2}\right) \ldots p_{N_{k}, i_{k}}\left(x_{k}\right) f\left(\frac{i_{1}}{N_{1}}, \ldots, \frac{i_{k}}{N_{k}}\right)}{\prod_{\lambda=1}^{k}\left(\vee_{i_{\lambda}=0}^{N_{\lambda}} p_{N_{\lambda}, i_{\lambda}}\left(x_{\lambda}\right)\right)},
\end{gathered}
$$

for all $x \in[0,1]^{k}$, by the maximum multiplicative principle, see (20). 
We make

Remark 9. The coordinate Max-product Bernstein operators are defined as follows $(\lambda=1, \ldots, k)$ :

$$
B_{N_{\lambda}}^{(M)}(g)\left(x_{\lambda}\right):=\frac{\vee_{i_{\lambda}=0}^{N_{\lambda}} p_{N_{\lambda}, i_{\lambda}}\left(x_{\lambda}\right) g\left(\frac{i_{\lambda}}{N_{\lambda}}\right)}{\vee_{i_{\lambda}=0}^{N_{\lambda}} p_{N_{\lambda}, i_{\lambda}}\left(x_{\lambda}\right)},
$$

for all $N_{\lambda} \in \mathbb{N}, x_{\lambda} \in[0,1]$, and $g \in C_{+}([0,1]):=\left\{g:[0,1] \rightarrow \mathbb{R}_{+}\right.$continuous $\}$.

Here we have

$$
p_{N_{\lambda}, i_{\lambda}}\left(x_{\lambda}\right)=\left(\begin{array}{c}
N_{\lambda} \\
i_{\lambda}
\end{array}\right) x_{\lambda}^{i_{\lambda}}\left(1-x_{\lambda}\right)^{N_{\lambda}-i_{\lambda}}, \text { for all } \lambda=1, \ldots, k ; x_{\lambda} \in[0,1] .
$$

In case $f \in C_{+}\left([0,1]^{k}\right)$ is such that $f(x):=g\left(x_{\lambda}\right)$, for all $x \in[0,1]^{k}$, where $x=\left(x_{1}, \ldots, x_{\lambda}, \ldots, x_{k}\right)$ and $g \in C_{+}([0,1])$, we get that

$$
B_{\vec{N}}^{(M)}(f)(x)=B_{N_{\lambda}}^{(M)}(g)\left(x_{\lambda}\right),
$$

by the maximum multiplicative principle (20) and simplification of (25).

Clearly it holds that

$$
B_{\vec{N}}^{(M)}(f)(x)=f(x), \forall x=\left(x_{1}, \ldots, x_{k}\right) \in[0,1]^{k}: x_{\lambda} \in\{0,1\}, \lambda=1, \ldots, k .
$$

We present

Theorem 10. Let $[0,1]^{k}, k \in \mathbb{N}-\{1\}$ with the $l_{1}$-norm and let $x=\left(x_{1}, \ldots, x_{k}\right) \in(0,1)^{k}$ be fixed. Let $f \in$ $C^{n}\left([0,1]^{k}, \mathbb{R}_{+}\right), n \in \mathbb{N}$, and suppose that each nth partial derivative $f_{\alpha}=\frac{\partial^{\alpha} f}{\partial x^{\alpha}}$, where $\alpha=\left(\alpha_{1}, \ldots, \alpha_{k}\right), \alpha_{i} \geq 0$, $i=1, \ldots, k$, and $|\alpha|=\sum_{i=1}^{k} \alpha_{i}=n$ has, relative to $[0,1]^{k}$ and the $l_{1}$-norm, a modulus of continuity $\omega_{1}\left(f_{\alpha}, \cdot\right)$, and that each $\left|f_{\alpha}(t)\right|$ is a convex function of $t \in[0,1]^{k}$, for all $\alpha:|\alpha|=n$. Assume further that $f_{\alpha}(x)=0$, for all $\alpha$ : $|\alpha|=1, \ldots, n$, and the ball in $\mathbb{R}^{k}: B\left(x, \frac{1}{\sqrt{N_{\min }^{\star}+1}}\right) \subset[0,1]^{k}$, for a sufficiently large $N_{\min }^{\star}:=\min \left\{N_{1}^{\star}, \ldots, N_{k}^{\star}\right\}$, where $\vec{N}^{\star}:=\left(N_{1}^{\star}, \ldots, N_{k}^{\star}\right) \in \mathbb{N}^{k}$. Then

$$
\left|B_{\vec{N}}^{(M)}(f)(x)-f(x)\right| \leq\left(\frac{6 k^{n+1}}{(n+1) !}\right)\left(\max _{\alpha:|\alpha|=n} \omega_{1}\left(f_{\alpha}, \frac{1}{\sqrt{N_{\min }+1}}\right)\right),
$$

for all $\vec{N} \in \mathbb{N}^{k}$, where $\vec{N}:=\left(N_{1}, \ldots, N_{k}\right) \in \mathbb{N}^{k}$ and $N_{\min }:=\min \left\{N_{1}, \ldots, N_{k}\right\}$, with $N_{\min } \geq N_{\min }^{\star}$.

It holds that $\lim _{\vec{N} \rightarrow(\infty, \ldots, \infty)} B_{\vec{N}}^{(M)}(f)(x)=f(x)$.

Proof. By (19) we get:

$$
\begin{gathered}
\left|B_{\vec{N}}^{(M)}(f)(x)-f(x)\right| \stackrel{(28)}{\leq} \frac{\left(\max _{\alpha:|\alpha|=n} \omega_{1}\left(f_{\alpha}, h\right)\right) k^{n}}{h(n+1) !} \cdot \\
\left(\sum_{i=1}^{k} B_{N_{i}}^{(M)}\left(\left|t_{i}-x_{i}\right|^{n+1}\right)\left(x_{i}\right)\right) \stackrel{(23)}{\leq} \frac{\left(\max _{\alpha:|\alpha|=n} \omega_{1}\left(f_{\alpha}, h\right)\right) k^{n}}{h(n+1) !}\left(\sum_{i=1}^{k} \frac{6}{\sqrt{N_{i}+1}}\right) \leq \\
\quad\left(\frac{\left.\max _{\alpha:|\alpha|=n} \omega_{1}\left(f_{\alpha}, h\right)\right) k^{n+1}}{h(n+1) !}\left(\frac{6}{\sqrt{N_{\min }+1}}\right)=\left(\operatorname{setting} h:=\frac{1}{\sqrt{N_{\min }+1}}\right)\right. \\
\left(\max _{\alpha:|\alpha|=n} \omega_{1}\left(f_{\alpha}, \frac{1}{\sqrt{N_{\min }+1}}\right)\right),
\end{gathered}
$$

proving the claim. 
We need

Theorem 11. Let $Q$ with the $l_{1}$-norm $\|\cdot\|$, be a compact and convex subset of $\mathbb{R}^{k}, k \in \mathbb{N}-\{1\}$, and $f \in C_{+}(Q)$ : $|f(t)-f(x)|$ is a convex function in $t:=\left(t_{1}, \ldots, t_{k}\right) \in Q$ for a fixed $x=\left(x_{1}, \ldots, x_{k}\right) \in Q^{o}$. We denote $\omega_{1}(f, h):=$ $\sup _{x, y \in Q:}|f(x)-f(y)|, h>0$, the first modulus of continuity of $f$. We assume that the ball in $\mathbb{R}^{k}: B(x, h) \subset Q$. Let $x, y \in Q:$

$\|x-y\| \leq h$

$\left\{L_{N}\right\}_{N \in \mathbb{N}}$ be positive sublinear operators from $C_{+}(Q)$ into $C_{+}(Q), L_{N}(1)=1$, for all $N \in \mathbb{N}$. Then

$$
\begin{gathered}
\left|L_{N}(f)(x)-f(x)\right| \leq \frac{\omega_{1}(f, h)}{h} L_{N}(\|t-x\|)(x) \leq \\
\frac{\omega_{1}(f, h)}{h}\left(\sum_{i=1}^{k} L_{N}\left(\left|t_{i}-x_{i}\right|\right)(x)\right),
\end{gathered}
$$

for all $N \in \mathbb{N}$.

Proof. By Lemma 8.1.1 [2, p. 243], we get that:

$$
|f(t)-f(x)| \leq \frac{\omega_{1}(f, h)}{h}\|t-x\|, \forall t \in Q .
$$

By (18) we have

$$
\begin{gathered}
\left|L_{N}(f)(x)-f(x)\right| \leq L_{N}(|f(t)-f(x)|)(x) \leq \\
\frac{\omega_{1}(f, h)}{h} L_{N}(\|t-x\|)(x) \leq \frac{\omega_{1}(f, h)}{h}\left(\sum_{i=1}^{k} L_{N}\left(\left|t_{i}-x_{i}\right|\right)(x)\right),
\end{gathered}
$$

proving the claim.

We give

Theorem 12. Let $[0,1]^{k}, k \in \mathbb{N}-\{1\}$, with the $l_{1}$-norm $\|\cdot\|$ and $f \in C_{+}\left([0,1]^{k}\right):|f(t)-f(x)|$ is a convex function in $t \in[0,1]^{k}$ for a fixed $x \in(0,1)^{k}$. Assume that the ball in $\mathbb{R}^{k}: B\left(x, \frac{1}{\sqrt{N_{\min }^{*}+1}}\right) \subset[0,1]^{k}$, for a sufficiently large $N_{\min }^{\star}:=\min \left\{N_{1}^{\star}, \ldots, N_{k}^{\star}\right\}$, where $\vec{N}^{\star}:=\left(N_{1}^{\star}, \ldots, N_{k}^{\star}\right) \in \mathbb{N}^{k}$. Then

$$
\left|B_{\vec{N}}^{(M)}(f)(x)-f(x)\right| \leq 6 k \omega_{1}\left(f, \frac{1}{\sqrt{N_{\min }+1}}\right),
$$

for all $\vec{N} \in \mathbb{N}^{k}$, where $\vec{N}:=\left(N_{1}, \ldots, N_{k}\right) \in \mathbb{N}^{k}$ and $N_{\min }:=\min \left\{N_{1}, \ldots, N_{k}\right\}$, with $N_{\min } \geq N_{\min }^{\star}$.

It holds that $\lim _{\vec{N} \rightarrow(\infty, \ldots, \infty)} B_{\vec{N}}^{(M)}(f)(x)=f(x)$.

Proof. By (33) we have that

$$
\begin{gathered}
\left|B_{\vec{N}}^{(M)}(f)(x)-f(x)\right| \stackrel{(28)}{\leq} \frac{\omega_{1}(f, h)}{h}\left(\sum_{i=1}^{k} B_{N_{i}}^{(M)}\left(\left|t_{i}-x_{i}\right|\right)\left(x_{i}\right)\right) \\
\stackrel{(22)}{\leq} \frac{\omega_{1}(f, h)}{h}\left(\sum_{i=1}^{k} \frac{6}{\sqrt{N_{i}+1}}\right) \leq \frac{\omega_{1}(f, h)}{h}\left(\frac{6 k}{\sqrt{N_{\min }+1}}\right)
\end{gathered}
$$

(choosing $\left.h:=\frac{1}{\sqrt{N_{\min }+1}}\right)$

$$
=6 k \omega_{1}\left(f, \frac{1}{\sqrt{N_{\min }+1}}\right) \text {, }
$$

proving the claim. 
We continue with

Definition 13. [1, p.123] We define the bivariate Max-product Bernstein type operators:

$$
\bar{T}_{N}^{(M)}(f)(x, y):=\frac{\vee_{i=0}^{N} \vee_{j=0}^{N-i}\left(\begin{array}{c}
N \\
i
\end{array}\right)\left(\begin{array}{c}
N-i \\
j
\end{array}\right) x^{i} y^{j}(1-x-y)^{N-i-j} f\left(\frac{i}{N}, \frac{j}{N}\right)}{\vee_{i=0}^{N} \vee_{j=0}^{N-i}\left(\begin{array}{c}
N \\
i
\end{array}\right)\left(\begin{array}{c}
N-i \\
j
\end{array}\right) x^{i} y^{j}(1-x-y)^{N-i-j}},
$$

for all $(x, y) \in \Delta:=\{(x, y): x \geq 0, y \geq 0, x+y \leq 1\}, N \in \mathbb{N}$, and $f \in C_{+}(\Delta)$.

Remark 14. By [1, p. 137], Theorem 2.7.5 there, $\bar{T}_{N}^{(M)}$ is a positive sublinear operator mapping $C_{+}(\Delta)$ into itself and $\bar{T}_{N}^{(M)}(1)=1$, furthermore it holds that

$$
\left|\bar{T}_{N}^{(M)}(f)-\bar{T}_{N}^{(M)}(g)\right| \leq \bar{T}_{N}^{(M)}(|f-g|), \forall f, g \in C_{+}(\Delta), \forall N \in \mathbb{N} .
$$

By [1, p. 125] we get that $\bar{T}_{N}^{(M)}(f)(1,0)=f(1,0), \bar{T}_{N}^{(M)}(f)(0,1)=f(0,1)$, and $\bar{T}_{N}^{(M)}(f)(0,0)=f(0,0)$.

By [1, p.139], we have that $((x, y) \in \Delta)$ :

$$
\bar{T}_{N}^{(M)}(|\cdot-x|)(x, y)=B_{N}^{(M)}(|\cdot-x|)(x),
$$

and

$$
\bar{T}_{N}^{(M)}(|\cdot-y|)(x, y)=B_{N}^{(M)}(|\cdot-y|)(y) .
$$

Working in exactly the same way as (40), (41) are proved we also derive $(m \in \mathbb{N},(x, y) \in \Delta)$ :

$$
\bar{T}_{N}^{(M)}\left(|\cdot-x|^{m}\right)(x, y)=B_{N}^{(M)}\left(|\cdot-x|^{m}\right)(x),
$$

and

$$
\bar{T}_{N}^{(M)}\left(|\cdot-y|^{m}\right)(x, y)=B_{N}^{(M)}\left(|\cdot-y|^{m}\right)(y) .
$$

We present

Theorem 15. Let $\Delta \subset \mathbb{R}^{2}$ be endowed with the $l_{1}$-norm. Let $x:=\left(x_{1}, x_{2}\right) \in \Delta^{o}$ be fixed, and $f \in C^{n}\left(\Delta, \mathbb{R}_{+}\right)$, $n \in \mathbb{N}$. We assume that $f_{\alpha}(x)=0$, for all $\alpha:|\alpha|=1, \ldots, n$, and $\left|f_{\alpha}\right|$ is a convex function for all $\alpha:|\alpha|=n$. For a sufficiently large $N^{\star} \in \mathbb{N}$ we have that the disc in $\mathbb{R}^{2}: D\left(x, \frac{1}{\sqrt{N^{*}+1}}\right) \subset \Delta$. Then

$$
\left|\bar{T}_{N}^{(M)}(f)\left(x_{1}, x_{2}\right)-f\left(x_{1}, x_{2}\right)\right| \leq \frac{3 \cdot 2^{n+2}\left(\max _{\alpha:|\alpha|=n} \omega_{1}\left(f_{\alpha}, \frac{1}{\sqrt{N+1}}\right)\right)}{(n+1) !},
$$

for all $N \geq N^{\star}, N \in \mathbb{N}$.

$$
\text { It holds that } \lim _{N \rightarrow \infty} \bar{T}_{N}^{(M)}(f)\left(x_{1}, x_{2}\right)=f\left(x_{1}, x_{2}\right) \text {. }
$$

Proof. By (19) we get (here $\left.x:=\left(x_{1}, x_{2}\right) \in \Delta\right)$ :

$$
\begin{gathered}
\left|\bar{T}_{N}^{(M)}(f)\left(x_{1}, x_{2}\right)-f\left(x_{1}, x_{2}\right)\right| \leq \frac{\left(\max _{\alpha:|\alpha|=n} \omega_{1}\left(f_{\alpha}, h\right)\right) 2^{n}}{h(n+1) !} . \\
\left(\sum_{i=1}^{2} \bar{T}_{N}^{(M)}\left(\left|t_{i}-x_{i}\right|^{n+1}\right)(x)\right) \stackrel{(\operatorname{by}(42),(43))}{=} \\
\frac{\left(\max _{\alpha:|\alpha|=n} \omega_{1}\left(f_{\alpha}, h\right)\right) 2^{n}}{h(n+1) !}\left(\sum_{i=1}^{2} B_{N}^{(M)}\left(\left|t_{i}-x_{i}\right|^{n+1}\right)\left(x_{i}\right)\right)^{(23)}
\end{gathered}
$$




$$
\begin{gathered}
\frac{\left(\max _{\alpha:|\alpha|=n} \omega_{1}\left(f_{\alpha}, h\right)\right) 2^{n+1}}{h(n+1) !} \frac{6}{\sqrt{N+1}}=\frac{3 \cdot 2^{n+2}\left(\max _{\alpha:|\alpha|=n} \omega_{1}\left(f_{\alpha}, h\right)\right)}{(n+1) ! h \sqrt{N+1}}= \\
\quad\left(\operatorname{setting} h:=\frac{1}{\sqrt{N+1}}\right) \\
\frac{3 \cdot 2^{n+2}\left(\max _{\alpha:|\alpha|=n} \omega_{1}\left(f_{\alpha}, \frac{1}{\sqrt{N+1}}\right)\right)}{(n+1) !},
\end{gathered}
$$

proving the claim.

It follows:

Theorem 16. Let $\Delta \subset \mathbb{R}^{2}$ be endowed with the $l_{1}$-norm. Let $f \in C_{+}(\Delta)$. We assume that $|f(t)-f(x)|$ is convex in $t \in \Delta$ for a fixed $x \in \Delta^{o}$. For a sufficiently large $N^{\star} \in \mathbb{N}$ we have that the disc in $\mathbb{R}^{2}: D\left(x, \frac{1}{\sqrt{N^{\star}+1}}\right) \subset \Delta$. Then

$$
\left|\bar{T}_{N}^{(M)}(f)\left(x_{1}, x_{2}\right)-f\left(x_{1}, x_{2}\right)\right| \leq 12 \omega_{1}\left(f, \frac{1}{\sqrt{N+1}}\right)
$$

for all $N \geq N^{\star}, N \in \mathbb{N}$.

$$
\text { It holds that } \lim _{N \rightarrow \infty} \bar{T}_{N}^{(M)}(f)\left(x_{1}, x_{2}\right)=f\left(x_{1}, x_{2}\right) \text {. }
$$

Proof. By (33) we have

$$
\begin{aligned}
& \left|\bar{T}_{N}^{(M)}(f)\left(x_{1}, x_{2}\right)-f\left(x_{1}, x_{2}\right)\right| \leq \frac{\omega_{1}(f, h)}{h}\left(\sum_{i=1}^{2} \bar{T}_{N}^{(M)}\left(\left|t_{i}-x_{i}\right|\right)(x)\right) \\
& \text { (by (40), (41)) } \frac{\omega_{1}(f, h)}{h}\left(\sum_{i=1}^{2} B_{N}^{(M)}\left(\left|t_{i}-x_{i}\right|\right)\left(x_{i}\right)\right) \stackrel{(22)}{\leq} \\
& \frac{2 \omega_{1}(f, h)}{h} \frac{6}{\sqrt{N+1}}=\frac{12 \omega_{1}(f, h)}{h \sqrt{N+1}}=
\end{aligned}
$$

(setting $\left.h:=\frac{1}{\sqrt{N+1}}\right)$

$$
12 \omega_{1}\left(f, \frac{1}{\sqrt{N+1}}\right)
$$

proving the claim.

We make

Remark 17. The Max-product truncated Favard-Szász-Mirakjan operators

$$
T_{N}^{(M)}(f)(x)=\frac{\bigvee_{k=0}^{N} s_{N, k}(x) f\left(\frac{k}{N}\right)}{\bigvee_{k=0}^{N} s_{N, k}(x)}, x \in[0,1], N \in \mathbb{N}, f \in C_{+}([0,1]),
$$

$s_{N, k}(x)=\frac{(N x)^{k}}{k !}$, see also [[1]

By [1,pp. 178-179], we get that

$$
T_{N}^{(M)}(|\cdot-x|)(x) \leq \frac{3}{\sqrt{N}}, \forall x \in[0,1], \forall N \in \mathbb{N} .
$$

And from [3] we have

$$
T_{N}^{(M)}\left(|\cdot-x|^{m}\right)(x) \leq \frac{3}{\sqrt{N}}, \forall x \in[0,1], \forall N, m \in \mathbb{N} .
$$

We make 
Definition 18. Let $f \in C_{+}\left([0,1]^{k}\right), k \in \mathbb{N}-\{1\}$, and $\vec{N}=\left(N_{1}, \ldots, N_{k}\right) \in \mathbb{N}^{k}$. We define the multivariate Max-product truncated Favard-Szász-Mirakjan operators as follows:

$$
\begin{gathered}
T_{\vec{N}}^{(M)}(f)(x):= \\
\frac{\vee_{i_{1}=0}^{N_{1}} \vee_{i_{2}=0}^{N_{2}} \ldots \vee_{i_{k}=0}^{N_{k}} s_{N_{1}, i_{1}}\left(x_{1}\right) s_{N_{2}, i_{2}}\left(x_{2}\right) \ldots s_{N_{k}, i_{k}}\left(x_{k}\right) f\left(\frac{i_{1}}{N_{1}}, \ldots, \frac{i_{k}}{N_{k}}\right)}{\vee_{i_{1}=0}^{N_{1}} \vee_{i_{2}=0}^{N_{2}} \ldots \vee_{i_{k}=0}^{N_{k}} s_{N_{1}, i_{1}}\left(x_{1}\right) s_{N_{2}, i_{2}}\left(x_{2}\right) \ldots s_{N_{k}, i_{k}}\left(x_{k}\right)},
\end{gathered}
$$

for all $x=\left(x_{1}, \ldots, x_{k}\right) \in[0,1]^{k}$. Call $N_{\min }:=\min \left\{N_{1}, \ldots, N_{k}\right\}$.

The operators $T_{\vec{N}}^{(M)}(f)(x)$ are positive sublinear mapping $C_{+}\left([0,1]^{k}\right)$ into itself, and $T_{\vec{N}}^{(M)}(1)=1$.

We also have

$$
\begin{gathered}
T_{\vec{N}}^{(M)}(f)(x):= \\
\frac{\vee_{i_{1}=0}^{N_{1}} \vee_{i_{2}=0}^{N_{2}} \ldots \vee_{i_{k}=0}^{N_{k}} s_{N_{1}, i_{1}}\left(x_{1}\right) s_{N_{2}, i_{2}}\left(x_{2}\right) \ldots s_{N_{k}, i_{k}}\left(x_{k}\right) f\left(\frac{i_{1}}{N_{1}}, \ldots, \frac{i_{k}}{N_{k}}\right)}{\prod_{\lambda=1}^{k}\left(\vee_{i_{\lambda}=0}^{N_{\lambda}} s_{N_{\lambda}, i_{\lambda}}\left(x_{\lambda}\right)\right)},
\end{gathered}
$$

for all $x \in[0,1]^{k}$, by the maximum multiplicative principle, see (20).

We make

Remark 19. The coordinate Max-product truncated Favard-Szász-Mirakjan operators are defined as follows $(\lambda=1, \ldots, k)$ :

$$
T_{N_{\lambda}}^{(M)}(g)\left(x_{\lambda}\right):=\frac{\vee_{i_{\lambda}=0}^{N_{\lambda}} s_{N_{\lambda}, i_{\lambda}}\left(x_{\lambda}\right) g\left(\frac{i_{\lambda}}{N_{\lambda}}\right)}{\vee_{i_{\lambda}=0}^{N_{\lambda}} s_{N_{\lambda}, i_{\lambda}}\left(x_{\lambda}\right)},
$$

for all $N_{\lambda} \in \mathbb{N}, x_{\lambda} \in[0,1]$, and $g \in C_{+}([0,1])$.

Here we have

$$
s_{N_{\lambda}, i_{\lambda}}\left(x_{\lambda}\right)=\frac{\left(N_{\lambda} x_{\lambda}\right)^{i_{\lambda}}}{i_{\lambda} !}, \lambda=1, \ldots, k ; x_{\lambda} \in[0,1] .
$$

In case of $f \in C_{+}\left([0,1]^{k}\right)$ such that $f(x):=g\left(x_{\lambda}\right), \forall x \in[0,1]^{k}$, where $x=\left(x_{1}, \ldots, x_{\lambda}, \ldots, x_{k}\right)$ and $g \in$ $C_{+}([0,1])$, we get that

$$
T_{\vec{N}}^{(M)}(f)(x)=T_{N_{\lambda}}^{(M)}(g)\left(x_{\lambda}\right),
$$

by the maximum multiplicative principle (20) and simplification of (54).

We present

Theorem 20. Let $[0,1]^{k}, k \in \mathbb{N}-\{1\}$ with the $l_{1}$-norm and let $x=\left(x_{1}, \ldots, x_{k}\right) \in(0,1)^{k}$ be fixed. Let $f \in$ $C^{n}\left([0,1]^{k}, \mathbb{R}_{+}\right), n \in \mathbb{N}$, and suppose that each $n$th partial derivative $f_{\alpha}=\frac{\partial^{\alpha} f}{\partial x^{\alpha}}$, where $\alpha=\left(\alpha_{1}, \ldots, \alpha_{k}\right), \alpha_{i} \geq 0$, $i=1, \ldots, k$, and $|\alpha|=\sum_{i=1}^{k} \alpha_{i}=n$, has relative to $[0,1]^{k}$ and the $l_{1}$-norm a modulus of continuity $\omega_{1}\left(f_{\alpha}, \cdot\right)$, and that each $\left|f_{\alpha}(t)\right|$ is a convex function of $t \in[0,1]^{k}$, all $\alpha:|\alpha|=n$. Assume further that $f_{\alpha}(x)=0$, for all $\alpha:|\alpha|=1, \ldots, n$, and the ball in $\mathbb{R}^{k}: B\left(x, \frac{1}{\sqrt{N_{\min }^{\star}}}\right) \subset[0,1]^{k}$, for a sufficiently large $N_{\min }^{\star}:=\min \left\{N_{1}^{\star}, \ldots, N_{k}^{\star}\right\}$, where $\vec{N}^{\star}:=\left(N_{1}^{\star}, \ldots, N_{k}^{\star}\right) \in \mathbb{N}^{k}$. Then

$$
\left|T_{\vec{N}}^{(M)}(f)(x)-f(x)\right| \leq\left(\frac{3 k^{n+1}}{(n+1) !}\right)\left(\max _{\alpha:|\alpha|=n} \omega_{1}\left(f_{\alpha}, \frac{1}{\sqrt{N_{\min }}}\right)\right),
$$

for all $\vec{N} \in \mathbb{N}^{k}$, where $\vec{N}:=\left(N_{1}, \ldots, N_{k}\right) \in \mathbb{N}^{k}$ and $N_{\min }:=\min \left\{N_{1}, \ldots, N_{k}\right\}$, with $N_{\min } \geq N_{\min }^{\star}$.

It holds that $\lim _{\vec{N} \rightarrow(\infty, \ldots, \infty)} T_{\vec{N}}^{(M)}(f)(x)=f(x)$. 
Proof. By (19) we get:

$$
\begin{gathered}
\left|T_{\vec{N}}^{(M)}(f)(x)-f(x)\right| \stackrel{(57)}{\leq} \frac{\left(\max _{\alpha:|\alpha|=n} \omega_{1}\left(f_{\alpha}, h\right)\right) k^{n}}{h(n+1) !}\left(\sum_{i=1}^{k} T_{N_{i}}^{(M)}\left(\left|t_{i}-x_{i}\right|^{n+1}\right)\left(x_{i}\right)\right) \\
\stackrel{(52)}{\leq} \frac{\left(\max _{\alpha:|\alpha|=n} \omega_{1}\left(f_{\alpha}, h\right)\right) k^{n}}{h(n+1) !}\left(\sum_{i=1}^{k} \frac{3}{\sqrt{N_{i}}}\right) \leq \frac{3 k^{n+1}\left(\max _{\alpha:|\alpha|=n} \omega_{1}\left(f_{\alpha}, h\right)\right)}{h(n+1) ! \sqrt{N_{\min }}}=
\end{gathered}
$$

(setting $\left.h:=\frac{1}{\sqrt{N_{\min }}}\right)$

$$
\frac{3 k^{n+1}\left(\max _{\alpha:|\alpha|=n} \omega_{1}\left(f_{\alpha}, \frac{1}{\sqrt{N_{\min }}}\right)\right)}{(n+1) !},
$$

proving the claim.

It follows

Theorem 21. Let $[0,1]^{k}, k \in \mathbb{N}-\{1\}$ with the $l_{1}$-norm $\|\cdot\|$ and $f \in C_{+}\left([0,1]^{k}\right):|f(t)-f(x)|$ is a convex function in $t \in[0,1]^{k}$ for a fixed $x \in(0,1)^{k}$. Assume that the ball in $\mathbb{R}^{k}: B\left(x, \frac{1}{\sqrt{N_{\min }^{*}}}\right) \subset[0,1]^{k}$, for a sufficiently large $N_{\text {min }}^{\star}:=\min \left\{N_{1}^{\star}, \ldots, N_{k}^{\star}\right\}$; where $\vec{N}^{\star}:=\left(N_{1}^{\star}, \ldots, N_{k}^{\star}\right) \in \mathbb{N}^{k}$. Then

$$
\left|T_{\vec{N}}^{(M)}(f)(x)-f(x)\right| \leq 3 k \omega_{1}\left(f, \frac{1}{\sqrt{N_{\min }}}\right),
$$

for all $\vec{N} \in \mathbb{N}^{k}$, where $\vec{N}:=\left(N_{1}, \ldots, N_{k}\right) \in \mathbb{N}^{k}$ and $N_{\min }:=\min \left\{N_{1}, \ldots, N_{k}\right\}$, with $N_{\min } \geq N_{\text {min }}^{\star}$.

It holds that $\lim _{\vec{N} \rightarrow(\infty, \ldots, \infty)} T_{\vec{N}}^{(M)}(f)(x)=f(x)$.

Proof. By (33) we have that

$$
\begin{gathered}
\left|T_{\vec{N}}^{(M)}(f)(x)-f(x)\right| \stackrel{(57)}{\leq} \frac{\omega_{1}(f, h)}{h}\left(\sum_{i=1}^{k} T_{N_{i}}^{(M)}\left(\left|t_{i}-x_{i}\right|\right)\left(x_{i}\right)\right) \\
\stackrel{(52)}{\leq} \frac{\omega_{1}(f, h)}{h}\left(\sum_{i=1}^{k} \frac{3}{\sqrt{N_{i}}}\right) \leq \frac{\omega_{1}(f, h)}{h} \frac{3 k}{\sqrt{N_{\min }}}
\end{gathered}
$$

(choosing $h:=\frac{1}{\sqrt{N_{\min }}}$ )

$$
=3 k \omega_{1}\left(f, \frac{1}{\sqrt{N_{\min }}}\right) \text {, }
$$

proving the claim.

We make

Remark 22. We mention the truncated Max-product Baskakov operator (see [1, p. 11])

$$
U_{N}^{(M)}(f)(x)=\frac{\bigvee_{k=0}^{N} b_{N, k}(x) f\left(\frac{k}{N}\right)}{\bigvee_{k=0}^{N} b_{N, k}(x)}, x \in[0,1], f \in C_{+}([0,1]), \forall N \in \mathbb{N},
$$

where

$$
b_{N, k}(x)=\left(\begin{array}{c}
N+k-1 \\
k
\end{array}\right) \frac{x^{k}}{(1+x)^{N+k}} .
$$


From [1, pp. 217-218], we get $(x \in[0,1])$

$$
\left(U_{N}^{(M)}(|\cdot-x|)\right)(x) \leq \frac{12}{\sqrt{N+1}}, N \geq 2, N \in \mathbb{N} .
$$

And as in [3], we obtain $(m \in \mathbb{N})$

$$
\left(U_{N}^{(M)}\left(|\cdot-x|^{m}\right)\right)(x) \leq \frac{12}{\sqrt{N+1}}, N \geq 2, N \in \mathbb{N}, \forall x \in[0,1] .
$$

Definition 23. Let $f \in C_{+}\left([0,1]^{k}\right), k \in \mathbb{N}-\{1\}$, and $\vec{N}=\left(N_{1}, \ldots, N_{k}\right) \in \mathbb{N}^{k}$. We define the multivariate Max-product truncated Baskakov operators as follows:

$$
\begin{gathered}
U_{\vec{N}}^{(M)}(f)(x):= \\
\frac{\vee_{i_{1}=0}^{N_{1}} \vee_{i_{2}=0}^{N_{2}} \ldots \vee_{i_{k}=0}^{N_{k}} b_{N_{1}, i_{1}}\left(x_{1}\right) b_{N_{2}, i_{2}}\left(x_{2}\right) \ldots b_{N_{k}, i_{k}}\left(x_{k}\right) f\left(\frac{i_{1}}{N_{1}}, \ldots, \frac{i_{k}}{N_{k}}\right)}{\vee_{i_{1}=0}^{N_{1}} \vee_{i_{2}=0}^{N_{2}} \ldots \vee_{i_{k}=0}^{N_{k}} b_{N_{1}, i_{1}}\left(x_{1}\right) b_{N_{2}, i_{2}}\left(x_{2}\right) \ldots b_{N_{k}, i_{k}}\left(x_{k}\right)},
\end{gathered}
$$

for all $x=\left(x_{1}, \ldots, x_{k}\right) \in[0,1]^{k}$. Call $N_{\min }:=\min \left\{N_{1}, \ldots, N_{k}\right\}$.

The operators $U_{\vec{N}}^{(M)}(f)(x)$ are positive sublinear mapping $C_{+}\left([0,1]^{k}\right)$ into itself, and $U_{\vec{N}}^{(M)}(1)=1$.

We also have

$$
\begin{gathered}
U_{\vec{N}}^{(M)}(f)(x):= \\
\frac{\vee_{i_{1}=0}^{N_{1}} \vee_{i_{2}=0}^{N_{2}} \ldots \vee_{i_{k}=0}^{N_{k}} b_{N_{1}, i_{1}}\left(x_{1}\right) b_{N_{2}, i_{2}}\left(x_{2}\right) \ldots b_{N_{k}, i_{k}}\left(x_{k}\right) f\left(\frac{i_{1}}{N_{1}}, \ldots, \frac{i_{k}}{N_{k}}\right)}{\prod_{\lambda=1}^{k}\left(\vee_{i_{\lambda}=0}^{N_{\lambda}} b_{N_{\lambda}, i_{\lambda}}\left(x_{\lambda}\right)\right)},
\end{gathered}
$$

for all $x \in[0,1]^{k}$, by the maximum multiplicative principle, see (20).

We make

Remark 24. The coordinate Max-product truncated Baskakov operators are defined as follows $(\lambda=1, \ldots, k)$ :

$$
U_{N_{\lambda}}^{(M)}(g)\left(x_{\lambda}\right):=\frac{\vee_{i_{\lambda}=0}^{N_{\lambda}} b_{N_{\lambda}, i_{\lambda}}\left(x_{\lambda}\right) g\left(\frac{i_{\lambda}}{N_{\lambda}}\right)}{\vee_{i_{\lambda}=0}^{N_{\lambda}} b_{N_{\lambda}, i_{\lambda}}\left(x_{\lambda}\right)},
$$

for all $N_{\lambda} \in \mathbb{N}, x_{\lambda} \in[0,1]$, and $g \in C_{+}([0,1])$.

Here we have

$$
b_{N_{\lambda}, i_{\lambda}}\left(x_{\lambda}\right)=\left(\begin{array}{c}
N_{\lambda}+i_{\lambda}-1 \\
i_{\lambda}
\end{array}\right) \frac{x_{\lambda}^{i_{\lambda}}}{\left(1+x_{\lambda}\right)^{N+i_{\lambda}}}, \lambda=1, \ldots, k ; x_{\lambda} \in[0,1] .
$$

In case of $f \in C_{+}\left([0,1]^{k}\right)$ such that $f(x):=g\left(x_{\lambda}\right)$, for all $x \in[0,1]^{k}$, where $x=\left(x_{1}, \ldots, x_{\lambda}, \ldots, x_{k}\right)$ and $g \in C_{+}([0,1])$, we get that

$$
U_{\vec{N}}^{(M)}(f)(x)=U_{N_{\lambda}}^{(M)}(g)\left(x_{\lambda}\right),
$$

by the maximum multiplicative principle (20) and simplification of (68).

We present

Theorem 25. Let $[0,1]^{k}, k \in \mathbb{N}-\{1\}$ with the $l_{1}$-norm and let $x=\left(x_{1}, \ldots, x_{k}\right) \in(0,1)^{k}$ be fixed. Let $f \in$ $C^{n}\left([0,1]^{k}, \mathbb{R}_{+}\right), n \in \mathbb{N}$, and suppose that each $n$th partial derivative $f_{\alpha}=\frac{\partial^{\alpha} f}{\partial x^{\alpha}}$, where $\alpha=\left(\alpha_{1}, \ldots, \alpha_{k}\right), \alpha_{i} \geq 0$, $i=1, \ldots, k$, and $|\alpha|=\sum_{i=1}^{k} \alpha_{i}=n$ has, relative to $[0,1]^{k}$ and the $l_{1}$-norm, a modulus of continuity $\omega_{1}\left(f_{\alpha}, \cdot\right)$, and that each $\left|f_{\alpha}(t)\right|$ is a convex function of $t \in[0,1]^{k}$, all $\alpha:|\alpha|=n$. Assume further that $f_{\alpha}(x)=0$, for all $\alpha$ : 
$|\alpha|=1, \ldots, n$, and the ball in $\mathbb{R}^{k}: B\left(x, \frac{1}{\sqrt{N_{\min }^{*}+1}}\right) \subset[0,1]^{k}$, for a sufficiently large $N_{\min }^{\star}:=\min \left\{N_{1}^{\star}, \ldots, N_{k}^{\star}\right\}$, where $\vec{N}^{\star}:=\left(N_{1}^{\star}, \ldots, N_{k}^{\star}\right) \in \mathbb{N}^{k}$. Then

$$
\left|U_{\vec{N}}^{(M)}(f)(x)-f(x)\right| \leq\left(\frac{12 k^{n+1}}{(n+1) !}\right)\left(\max _{\alpha:|\alpha|=n} \omega_{1}\left(f_{\alpha}, \frac{1}{\sqrt{N_{\min }+1}}\right)\right),
$$

for all $\vec{N} \in(\mathbb{N}-\{1\})^{k}$, where $\vec{N}:=\left(N_{1}, \ldots, N_{k}\right) \in \mathbb{N}^{k}$ and $N_{\min }:=\min \left\{N_{1}, \ldots, N_{k}\right\}$, with $N_{\min } \geq N_{\min }^{\star}$.

It holds that $\lim _{\vec{N} \rightarrow(\infty, \ldots, \infty)} U_{\vec{N}}^{(M)}(f)(x)=f(x)$.

Proof. By (19) we get:

$$
\begin{aligned}
& \left|U_{\vec{N}}^{(M)}(f)(x)-f(x)\right| \stackrel{(71)}{\leq} \frac{\left(\max _{\alpha:|\alpha|=n} \omega_{1}\left(f_{\alpha}, h\right)\right) k^{n}}{h(n+1) !} . \\
& \left(\sum_{i=1}^{k} U_{N_{i}}^{(M)}\left(\left|t_{i}-x_{i}\right|^{n+1}\right)\left(x_{i}\right)\right)^{(66)} \\
& \frac{\left(\max _{\alpha:|\alpha|=n} \omega_{1}\left(f_{\alpha}, h\right)\right) k^{n}}{h(n+1) !}\left(\sum_{i=1}^{k} \frac{12}{\sqrt{N_{i}+1}}\right) \leq \frac{\left(\max _{\alpha:|\alpha|=n} \omega_{1}\left(f_{\alpha}, h\right)\right) k^{n+1}}{h(n+1) !} \frac{12}{\sqrt{N_{\min }+1}}=
\end{aligned}
$$

(setting $\left.h:=\frac{1}{\sqrt{N_{\min }+1}}\right)$

$$
\left(\frac{12 k^{n+1}}{(n+1) !}\right)\left(\max _{\alpha:|\alpha|=n} \omega_{1}\left(f_{\alpha}, \frac{1}{\sqrt{N_{\min }+1}}\right)\right)
$$

proving the claim.

It follows

Theorem 26. Let $[0,1]^{k}, k \in \mathbb{N}-\{1\}$, with the $l_{1}$-norm $\|\cdot\|$ and $f \in C_{+}\left([0,1]^{k}\right):|f(t)-f(x)|$ is a convex function in $t \in[0,1]^{k}$ for a fixed $x \in(0,1)^{k}$. Assume that the ball in $\mathbb{R}^{k}: B\left(x, \frac{1}{\sqrt{N_{\min }^{*}+1}}\right) \subset[0,1]^{k}$, for a sufficiently large $N_{\text {min }}^{\star}:=\min \left\{N_{1}^{\star}, \ldots, N_{k}^{\star}\right\}$, where $\vec{N}^{\star}:=\left(N_{1}^{\star}, \ldots, N_{k}^{\star}\right) \in \mathbb{N}^{k}$. Then

$$
\left|U_{\vec{N}}^{(M)}(f)(x)-f(x)\right| \leq 12 k \omega_{1}\left(f, \frac{1}{\sqrt{N_{\min }+1}}\right),
$$

for all $\vec{N} \in(\mathbb{N}-\{1\})^{k}$, where $\vec{N}:=\left(N_{1}, \ldots, N_{k}\right) \in \mathbb{N}^{k}$ and $N_{\min }:=\min \left\{N_{1}, \ldots, N_{k}\right\}$, with $N_{\min } \geq N_{\min }^{\star}$.

It holds that $\lim _{\vec{N} \rightarrow(\infty, \ldots, \infty)} U_{\vec{N}}^{(M)}(f)(x)=f(x)$.

Proof. By (33) we have that

$$
\begin{aligned}
& \left|U_{\vec{N}}^{(M)}(f)(x)-f(x)\right| \stackrel{(71)}{\leq} \frac{\omega_{1}(f, h)}{h}\left(\sum_{i=1}^{k} U_{N_{i}}^{(M)}\left(\left|t_{i}-x_{i}\right|\right)\left(x_{i}\right)\right) \\
& \stackrel{(65)}{\leq} \frac{\omega_{1}(f, h)}{h}\left(\sum_{i=1}^{k} \frac{12}{\sqrt{N_{i}+1}}\right) \leq \frac{\omega_{1}(f, h)}{h}\left(\frac{12 k}{\sqrt{N_{\min }+1}}\right)
\end{aligned}
$$

(choosing $h:=\frac{1}{\sqrt{N_{\min }+1}}$ )

$$
=12 k \omega_{1}\left(f, \frac{1}{\sqrt{N_{\min }+1}}\right),
$$

proving the claim. 
We make

Remark 27. Here we mention the Max-product truncated sampling operators (see [1, p. 13]) defined by

$$
W_{N}^{(M)}(f)(x):=\frac{\bigvee_{k=0}^{N} \frac{\sin (N x-k \pi)}{N x-k \pi} f\left(\frac{k \pi}{N}\right)}{\bigvee_{k=0}^{N} \frac{\sin (N x-k \pi)}{N x-k \pi}}, x \in[0, \pi],
$$

$f:[0, \pi] \rightarrow \mathbb{R}_{+}$, continuous,

and

$$
K_{N}^{(M)}(f)(x):=\frac{\bigvee_{k=0}^{N} \frac{\sin ^{2}(N x-k \pi)}{(N x-k \pi)^{2}} f\left(\frac{k \pi}{N}\right)}{\bigvee_{k=0}^{N} \frac{\sin ^{2}(N x-k \pi)}{(N x-k \pi)^{2}}}, x \in[0, \pi],
$$

$f:[0, \pi] \rightarrow \mathbb{R}_{+}$, continuous.

By convention we take $\frac{\sin (0)}{0}=1$, which implies for every $x=\frac{k \pi}{N}, k \in\{0,1, \ldots, N\}$ that we have $\frac{\sin (N x-k \pi)}{N x-k \pi}=$ 1.

We define the Max-product truncated combined sampling operators

$$
M_{N}^{(M)}(f)(x):=\frac{\bigvee_{k=0}^{N} \rho_{N, k}(x) f\left(\frac{k \pi}{N}\right)}{\bigvee_{k=0}^{N} \rho_{N, k}(x)}, x \in[0, \pi],
$$

$f \in C_{+}([0, \pi])$, where

$$
M_{N}^{(M)}(f)(x):=\left\{\begin{array}{l}
W_{N}^{(M)}(f)(x), \text { if } \rho_{N, k}(x):=\frac{\sin (N x-k \pi)}{N x-k \pi} \\
K_{N}^{(M)}(f)(x), \text { if } \rho_{N, k}(x):=\left(\frac{\sin (N x-k \pi)}{N x-k \pi}\right)^{2}
\end{array}\right.
$$

By [1, p. 346 and p. 352] we get

$$
\left(M_{N}^{(M)}(|\cdot-\chi|)\right)(x) \leq \frac{\pi}{2 N}
$$

and by $[4](m \in \mathbb{N})$ we have

$$
\left(M_{N}^{(M)}\left(|\cdot-x|^{m}\right)\right)(x) \leq \frac{\pi^{m}}{2 N}, \forall x \in[0, \pi], \forall N \in \mathbb{N} .
$$

We give

Definition 28. Let $f \in C_{+}\left([0, \pi]^{k}\right), k \in \mathbb{N}-\{1\}$, and $\vec{N}=\left(N_{1}, \ldots, N_{k}\right) \in \mathbb{N}^{k}$. We define the multivariate Max-product truncated combined sampling operators as follows:

$$
\begin{gathered}
M_{\vec{N}}^{(M)}(f)(x):= \\
\frac{\vee_{i_{1}=0}^{N_{1}} \vee_{i_{2}=0}^{N_{2}} \ldots \vee_{i_{k}=0}^{N_{k}} \rho_{N_{1}, i_{1}}\left(x_{1}\right) \rho_{N_{2}, i_{2}}\left(x_{2}\right) \ldots \rho_{N_{k}, i_{k}}\left(x_{k}\right) f\left(\frac{i_{1} \pi}{N_{1}}, \frac{i_{2} \pi}{N_{2}}, \ldots, \frac{i_{k} \pi}{N_{k}}\right)}{\vee_{i_{1}=0}^{N_{1}} \vee_{i_{2}=0}^{N_{2}} \ldots \vee_{i_{k}=0}^{N_{k}} \rho_{N_{1}, i_{1}}\left(x_{1}\right) \rho_{N_{2}, i_{2}}\left(x_{2}\right) \ldots \rho_{N_{k}, i_{k}}\left(x_{k}\right)},
\end{gathered}
$$

for all $x=\left(x_{1}, \ldots, x_{k}\right) \in[0, \pi]^{k}$. Call $N_{\min }:=\min \left\{N_{1}, \ldots, N_{k}\right\}$.

The operators $M_{\vec{N}}^{(M)}(f)(x)$ are positive sublinear mapping $C_{+}\left([0, \pi]^{k}\right)$ into itself, and $M_{\vec{N}}^{(M)}(1)=1$.

We also have

$$
\begin{gathered}
M_{\vec{N}}^{(M)}(f)(x):= \\
\frac{\vee_{i_{1}=0}^{N_{1}} \vee_{i_{2}=0}^{N_{2}} \ldots \vee_{i_{k}=0}^{N_{k}} \rho_{N_{1}, i_{1}}\left(x_{1}\right) \rho_{N_{2}, i_{2}}\left(x_{2}\right) \ldots \rho_{N_{k}, i_{k}}\left(x_{k}\right) f\left(\frac{i_{1} \pi}{N_{1}}, \frac{i_{2} \pi}{N_{2}}, \ldots, \frac{i_{k} \pi}{N_{k}}\right)}{\prod_{\lambda=1}^{k}\left(\vee_{i_{\lambda}=0}^{N_{\lambda}} \rho_{N_{\lambda}, i_{\lambda}}\left(x_{\lambda}\right)\right)},
\end{gathered}
$$

for all $x \in[0, \pi]^{k}$, by the maximum multiplicative principle, see (20). 
We make

Remark 29. The coordinate Max-product truncated combined sampling operators are defined as follows ( $\lambda=$ $1, \ldots, k)$ :

$$
M_{N_{\lambda}}^{(M)}(g)\left(x_{\lambda}\right):=\frac{\vee_{i_{\lambda}=0}^{N_{\lambda}} \rho_{N_{\lambda}, i_{\lambda}}\left(x_{\lambda}\right) g\left(\frac{i_{\lambda} \pi}{N_{\lambda}}\right)}{\vee_{i_{\lambda}=0}^{N_{\lambda}} \rho_{N_{\lambda}, i_{\lambda}}\left(x_{\lambda}\right)},
$$

for all $N_{\lambda} \in \mathbb{N}, x_{\lambda} \in[0, \pi]$, and $g \in C_{+}([0, \pi])$.

Here we have $\left(\lambda=1, \ldots, k ; x_{\lambda} \in[0, \pi]\right)$

$$
\rho_{N_{\lambda}, i_{\lambda}}\left(x_{\lambda}\right)=\left\{\begin{array}{l}
\frac{\sin \left(N_{\lambda} x_{\lambda}-i_{\lambda} \pi\right)}{N_{\lambda} x_{\lambda}-i_{\lambda} \pi}, \text { if } M_{N_{\lambda}}^{(M)}=W_{N_{\lambda}}^{(M)}, \\
\left(\frac{\sin \left(N_{\lambda} x_{\lambda}-i_{\lambda} \pi\right)}{N_{\lambda} x_{\lambda}-i_{\lambda} \pi}\right)^{2}, \text { if } M_{N_{\lambda}}^{(M)}=K_{N_{\lambda}}^{(M)} .
\end{array}\right\} .
$$

In case of $f \in C_{+}\left([0, \pi]^{k}\right)$ such that $f(x):=g\left(x_{\lambda}\right)$, for all $x \in[0, \pi]^{k}$, where $x=\left(x_{1}, \ldots, x_{\lambda}, \ldots, x_{k}\right)$ and $g \in C_{+}([0, \pi])$, we get that

$$
M_{\vec{N}}^{(M)}(f)(x)=M_{N_{\lambda}}^{(M)}(g)\left(x_{\lambda}\right),
$$

by the maximum multiplicative principle (20) and simplification of (84).

We present

Theorem 30. Let $[0, \pi]^{k}, k \in \mathbb{N}-\{1\}$ with the $l_{1}$-norm and let $x \in(0, \pi)^{k}$ be fixed. Let $f \in C^{n}\left([0, \pi]^{k}, \mathbb{R}_{+}\right)$, $n \in \mathbb{N}$, and suppose that each nth partial derivative $f_{\alpha}=\frac{\partial^{\alpha} f}{\partial x^{\alpha}}$, where $\alpha=\left(\alpha_{1}, \ldots, \alpha_{k}\right), \alpha_{i} \geq 0, i=1, \ldots, k$, and $|\alpha|=\sum_{i=1}^{k} \alpha_{i}=n$, has relative to $[0, \pi]^{k}$ and the $l_{1}$-norm a modulus of continuity $\omega_{1}\left(f_{\alpha}, \cdot\right)$, and that each $\left|f_{\alpha}(t)\right|$ is a convex function of $t \in[0, \pi]^{k}$, for all $\alpha:|\alpha|=n$. Assume further that $f_{\alpha}(x)=0$, for all $\alpha:|\alpha|=1, \ldots, n$, and the ball in $\mathbb{R}^{k}: B\left(x, \frac{1}{N_{\min }^{*}}\right) \subset[0, \pi]^{k}$, for a sufficiently large $N_{\min }^{\star}:=\min \left\{N_{1}^{\star}, \ldots, N_{k}^{\star}\right\}$, where $\vec{N}^{\star}:=\left(N_{1}^{\star}, \ldots, N_{k}^{\star}\right) \in \mathbb{N}^{k}$. Then

$$
\left|M_{\vec{N}}^{(M)}(f)(x)-f(x)\right| \leq \frac{(k \pi)^{n+1}}{2(n+1) !}\left(\max _{\alpha:|\alpha|=n} \omega_{1}\left(f_{\alpha}, \frac{1}{N_{\min }}\right)\right),
$$

for all $\vec{N} \in \mathbb{N}^{k}$, where $\vec{N}:=\left(N_{1}, \ldots, N_{k}\right) \in \mathbb{N}^{k}$ and $N_{\min }:=\min \left\{N_{1}, \ldots, N_{k}\right\}$, with $N_{\min } \geq N_{\min }^{\star}$.

It holds that $\underset{\vec{N} \rightarrow(\infty, \ldots, \infty)}{ } M_{\vec{N}}^{(M)}(f)(x)=f(x)$.

Proof. By (19) we get:

$$
\begin{gathered}
\left|M_{\vec{N}}^{(M)}(f)(x)-f(x)\right|_{\leq}^{(87)} \underset{\left(\max _{\alpha:|\alpha|=n} \omega_{1}\left(f_{\alpha}, h\right)\right) k^{n}}{h(n+1) !} \cdot \\
\left(\sum_{i=1}^{k} M_{N_{i}}^{(M)}\left(\left|t_{i}-x_{i}\right|^{n+1}\right)\left(x_{i}\right)\right)_{\leq}^{(82)} \\
\frac{\left(\max _{\alpha:|\alpha|=n} \omega_{1}\left(f_{\alpha}, h\right)\right) k^{n}}{h(n+1) !}\left(\sum_{i=1}^{k} \frac{\pi^{n+1}}{2 N_{i}}\right) \leq \frac{\left(\max _{\alpha:|\alpha|=n} \omega_{1}\left(f_{\alpha}, h\right)\right) k^{n+1} \pi^{n+1}}{2(n+1) ! h N_{\min }}=
\end{gathered}
$$

(setting $\left.h:=\frac{1}{N_{\min }}\right)$

$$
\frac{\left(\max _{\alpha:|\alpha|=n} \omega_{1}\left(f_{\alpha}, \frac{1}{N_{\min }}\right)\right)(k \pi)^{n+1}}{2(n+1) !},
$$

proving the claim.

We continue with 
Theorem 31. Let $[0, \pi]^{k}, k \in \mathbb{N}-\{1\}$, with the $l_{1}$-norm and $f \in C_{+}\left([0, \pi]^{k}\right):|f(t)-f(x)|$ is a convex function in $t \in[0, \pi]^{k}$ for a fixed $x \in(0, \pi)^{k}$. Assume that the ball in $\mathbb{R}^{k}: B\left(x, \frac{1}{N_{\min }^{*}}\right) \subset[0, \pi]^{k}$, for a sufficiently large $N_{\text {min }}^{\star}:=\min \left\{N_{1}^{\star}, \ldots, N_{k}^{\star}\right\}$, where $\vec{N}^{\star}:=\left(N_{1}^{\star}, \ldots, N_{k}^{\star}\right) \in \mathbb{N}^{k}$. Then

$$
\left|M_{\vec{N}}^{(M)}(f)(x)-f(x)\right| \leq\left(\frac{k \pi}{2}\right) \omega_{1}\left(f, \frac{1}{N_{\min }}\right),
$$

for all $\vec{N} \in \mathbb{N}^{k}$, where $\vec{N}:=\left(N_{1}, \ldots, N_{k}\right) \in \mathbb{N}^{k}$ and $N_{\min }:=\min \left\{N_{1}, \ldots, N_{k}\right\}$, with $N_{\min } \geq N_{\min }^{\star}$.

It holds that $\lim _{\vec{N} \rightarrow(\infty, \ldots, \infty)} M_{\vec{N}}^{(M)}(f)(x)=f(x)$.

Proof. By (33) we have that

$$
\begin{aligned}
& \left|M_{\vec{N}}^{(M)}(f)(x)-f(x)\right| \stackrel{(87)}{\leq} \frac{\omega_{1}(f, h)}{h}\left(\sum_{i=1}^{k} M_{N_{i}}^{(M)}\left(\left|t_{i}-x_{i}\right|\right)\left(x_{i}\right)\right) \\
& \stackrel{(81)}{\leq} \frac{\omega_{1}(f, h)}{h}\left(\sum_{i=1}^{k} \frac{\pi}{2 N_{i}}\right) \leq \frac{\omega_{1}(f, h)}{h} \frac{(k \pi)}{2 N_{\min }}=
\end{aligned}
$$

(setting $\left.h:=\frac{1}{N_{\min }}\right)$

$$
\left(\frac{k \pi}{2}\right) \omega_{1}\left(f, \frac{1}{N_{\min }}\right)
$$

proving the claim.

We make

Remark 32. Let $f \in C_{+}([-1,1])$. Let the Chebyshev knots of second kind $x_{N, k}=\cos \left(\left(\frac{N-k}{N-1}\right) \pi\right) \in[-1,1]$, $k=1, \ldots, N, N \in \mathbb{N}-\{1\}$, which are the roots of $\omega_{N}(x)=\sin (N-1) t \sin t, x=\cos t \in[-1,1]$. Notice that $x_{N, 1}=-1$ and $x_{N, N}=1$.

Define

$$
l_{N, k}(x):=\frac{(-1)^{k-1} \omega_{N}(x)}{\left(1+\delta_{k, 1}+\delta_{k, N}\right)(N-1)\left(x-x_{N, k}\right)},
$$

$N \geq 2, k=1, \ldots, N$, and $\omega_{N}(x)=\prod_{k=1}^{N}\left(x-x_{N, k}\right)$ and $\delta_{i, j}$ denotes the Kronecker's symbol, that is $\delta_{i, j}=1$, if $i=j$, and $\delta_{i, j}=0$, if $i \neq j$.

The Max-product Lagrange interpolation operators on Chebyshev knots of second kind, plus the endpoints \pm 1 , are defined by $[1, p .12]$

$$
L_{N}^{(M)}(f)(x)=\frac{\bigvee_{k=1}^{N} l_{N, k}(x) f\left(x_{N, k}\right)}{\bigvee_{k=1}^{N} l_{N, k}(x)}, x \in[-1,1]
$$

By [1, pp. 297-298] and [4], we get that

$$
L_{N}^{(M)}\left(|\cdot-x|^{m}\right)(x) \leq \frac{2^{m+1} \pi^{2}}{3(N-1)},
$$

for all $x \in(-1,1)$ and $m \in \mathbb{N}$; for all $N \in \mathbb{N}, N \geq 4$.

We see that $L_{N}^{(M)}(f)(x) \geq 0$ is well-defined and continuous for any $x \in[-1,1]$. Following [1, p. 289], because $\sum_{k=1}^{N} l_{N, k}(x)=1, \forall x \in[-1,1]$, for any $x$ there exists $k \in\{1, \ldots, N\}: l_{N, k}(x)>0$, hence $\bigvee_{k=1}^{N} l_{N, k}(x)>0$. We have that $l_{N, k}\left(x_{N, k}\right)=1$, and $l_{N, k}\left(x_{N, j}\right)=0$, if $k \neq j$. Furthermore, it holds that $L_{N}^{(M)}(f)\left(x_{N, j}\right)=f\left(x_{N, j}\right)$, for all $j \in\{1, \ldots, N\}$, and $L_{N}^{(M)}(1)=1$.

By [1, pp. 289-290], $L_{N}^{(M)}$ are positive sublinear operators.

We give 
Definition 33. Let $f \in C_{+}\left([-1,1]^{k}\right), k \in \mathbb{N}-\{1\}$, and $\vec{N}=\left(N_{1}, \ldots, N_{k}\right) \in(\mathbb{N}-\{1\})^{k}$. We define the multivariate Max-product Lagrange interpolation operators on Chebyshev knots of second kind, plus the endpoints \pm 1 , as follows:

$$
\begin{gathered}
L_{\vec{N}}^{(M)}(f)(x):= \\
\frac{\vee_{i_{1}=1}^{N_{1}} \vee_{i_{2}=1}^{N_{2}} \ldots \vee_{i_{k}=1}^{N_{k}} l_{N_{1}, i_{1}}\left(x_{1}\right) l_{N_{2}, i_{2}}\left(x_{2}\right) \ldots l_{N_{k}, i_{k}}\left(x_{k}\right) f\left(x_{N_{1}, i_{1}}, x_{N_{2}, i_{2}}, \ldots, x_{N_{k}, i_{k}}\right)}{\vee_{i_{1}=1}^{N_{1}} \vee_{i_{2}=1}^{N_{2}} \ldots \vee_{i_{k}=1}^{N_{k}} l_{N_{1}, i_{1}}\left(x_{1}\right) l_{N_{2}, i_{2}}\left(x_{2}\right) \ldots l_{N_{k}, i_{k}}\left(x_{k}\right)},
\end{gathered}
$$

for all $x=\left(x_{1}, \ldots, x_{k}\right) \in[-1,1]^{k}$. Call $N_{\min }:=\min \left\{N_{1}, \ldots, N_{k}\right\}$.

The operators $L_{\vec{N}}^{(M)}(f)(x)$ are positive sublinear mapping $C_{+}\left([-1,1]^{k}\right)$ into itself, and $L_{\vec{N}}^{(M)}(1)=1$.

We also have

$$
\begin{gathered}
L_{\vec{N}}^{(M)}(f)(x):= \\
\frac{\vee_{i_{1}=1}^{N_{1}} \vee_{i_{2}=1}^{N_{2}} \ldots \vee_{i_{k}=1}^{N_{k}} l_{N_{1}, i_{1}}\left(x_{1}\right) l_{N_{2}, i_{2}}\left(x_{2}\right) \ldots l_{N_{k}, i_{k}}\left(x_{k}\right) f\left(x_{N_{1}, i_{1}}, x_{N_{2}, i_{2}}, \ldots, x_{N_{k}, i_{k}}\right)}{\prod_{\lambda=1}^{k}\left(\vee_{i_{\lambda}=1}^{N_{\lambda}} l_{N_{\lambda}, i_{\lambda}}\left(x_{\lambda}\right)\right)},
\end{gathered}
$$

for all $x=\left(x_{1}, \ldots, x_{\lambda}, \ldots, x_{k}\right) \in[-1,1]^{k}$, by the maximum multiplicative principle, see (20). Notice that $L_{\vec{N}}^{(M)}(f)\left(x_{N_{1}, i_{1}}, \ldots, x_{N_{k}, i_{k}}\right)=f\left(x_{N_{1}, i_{1}}, \ldots, x_{N_{k}, i_{k}}\right)$. The last is also true if $x_{N_{1}, i_{1}}, \ldots, x_{N_{k}, i_{k}} \in\{-1,1\}$.

We make

Remark 34. The coordinate Max-product Lagrange interpolation operators on Chebyshev knots of second kind, plus the endpoints \pm 1 , are defined as follows $(\lambda=1, \ldots, k)$ :

$$
L_{N_{\lambda}}^{(M)}(g)\left(x_{\lambda}\right):=\frac{\vee_{i_{\lambda}=1}^{N_{\lambda}} l_{N_{\lambda}, i_{\lambda}}\left(x_{\lambda}\right) g\left(x_{N_{\lambda}, i_{\lambda}}\right)}{\vee_{i_{\lambda}=1}^{N_{\lambda}} l_{N_{\lambda}, i_{\lambda}}\left(x_{\lambda}\right)},
$$

for all $N_{\lambda} \in \mathbb{N}, N_{\lambda} \geq 2, x_{\lambda} \in[-1,1]$, and $g \in C_{+}([-1,1])$.

Here we have $\left(\lambda=1, \ldots, k ; x_{\lambda} \in[-1,1]\right)$

$$
l_{N_{\lambda}, i_{\lambda}}\left(x_{\lambda}\right)=\frac{(-1)^{i_{\lambda}-1} \omega_{N_{\lambda}}\left(x_{\lambda}\right)}{\left(1+\delta_{i_{\lambda}, 1}+\delta_{i_{\lambda}, N_{\lambda}}\right)\left(N_{\lambda}-1\right)\left(x_{\lambda}-x_{N_{\lambda}, i_{\lambda}}\right)},
$$

$N_{\lambda} \geq 2, i_{\lambda}=1, \ldots, N_{\lambda}$ and $\omega_{N_{\lambda}}\left(x_{\lambda}\right)=\prod_{i_{\lambda}=1}^{N_{\lambda}}\left(x_{\lambda}-x_{N_{\lambda}, i_{\lambda}}\right)$; where $x_{N_{\lambda}, i_{\lambda}}=\cos \left(\left(\frac{N_{\lambda}-i_{\lambda}}{N_{\lambda}-1}\right) \pi\right) \in[-1,1]$, $i_{\lambda}=1, \ldots, N_{\lambda}\left(N_{\lambda} \geq 2\right)$ are roots of $\omega_{N_{\lambda}}\left(x_{\lambda}\right)=\sin \left(N_{\lambda}-1\right) t_{\lambda} \sin t_{\lambda}, x_{\lambda}=\cos t_{\lambda}$. Notice that $x_{N_{\lambda}, 1}=-1$, $x_{N_{\lambda}, N_{\lambda}}=1$.

In case off $\in C_{+}\left([-1,1]^{k}\right)$ such that $f(x):=g\left(x_{\lambda}\right)$, for all $x \in[-1,1]^{k}$, where $x=\left(x_{1}, \ldots, x_{\lambda}, \ldots, x_{k}\right)$ and $g \in C_{+}([-1,1])$, we get that

$$
L_{\vec{N}}^{(M)}(f)(x)=L_{N_{\lambda}}^{(M)}(g)\left(x_{\lambda}\right),
$$

by the maximum multiplicative principle (20) and simplification of (96).

We present

Theorem 35. Let $x \in(-1,1)^{k}, k \in \mathbb{N}-\{1\}$, be fixed, and let $f \in C^{n}\left([-1,1]^{k}, \mathbb{R}_{+}\right), n \in \mathbb{N}$. We assume that $f_{\alpha}(x)=0$, for all $\alpha:|\alpha|=1, \ldots, n$. Here $\left|f_{\alpha}\right|$ is assumed to be convex over $[-1,1]^{k}$, all $\alpha:|\alpha|=n$. The set $[-1,1]^{k}, k \in \mathbb{N}-\{1\}$ is endowed with the $l_{1}$-norm and $\omega_{1}\left(f_{\alpha}, \cdot\right)$, all $\alpha:|\alpha|=n$ is with respect to $l_{1}$-norm. For a sufficiently large $N_{\min }^{\star}:=\min \left\{N_{1}^{\star}, \ldots, N_{k}^{\star}\right\}$, where $\vec{N}^{\star}:=\left(N_{1}^{\star}, \ldots, N_{k}^{\star}\right) \in \mathbb{N}^{k} ; N_{i}^{\star} \geq 4, i=1, \ldots, k$, the ball in $\mathbb{R}^{k}: B\left(x, \frac{1}{\left(N_{\min }^{*}-1\right)}\right) \subset[-1,1]^{k}$. Then

$$
\left|L_{\vec{N}}^{(M)}(f)(x)-f(x)\right| \leq\left(\frac{2^{n+2} k^{n+1} \pi^{2}}{3(n+1) !}\right)\left(\max _{\alpha:|\alpha|=n} \omega_{1}\left(f_{\alpha}, \frac{1}{\left(N_{\min }-1\right)}\right)\right),
$$


for all $\vec{N} \in \mathbb{N}^{k}$, where $\vec{N}:=\left(N_{1}, \ldots, N_{k}\right) \in \mathbb{N}^{k}$ and $N_{\min }:=\min \left\{N_{1}, \ldots, N_{k}\right\}$, with $N_{\min } \geq N_{\min }^{\star}$.

It holds that $\lim _{\vec{N} \rightarrow(\infty, \ldots, \infty)} L_{\vec{N}}^{(M)}(f)(x)=f(x)$.

Proof. By (19) we get:

$$
\begin{gathered}
\left|L_{\vec{N}}^{(M)}(f)(x)-f(x)\right|_{\leq}^{(99)} \frac{\left(\max _{\alpha:|\alpha|=n} \omega_{1}\left(f_{\alpha}, h\right)\right) k^{n}}{h(n+1) !} . \\
\left(\sum_{i=1}^{k} L_{N_{i}}^{(M)}\left(\left|t_{i}-x_{i}\right|^{n+1}\right)\left(x_{i}\right)\right) \stackrel{(94)}{\leq} \\
\frac{\left(\max _{\alpha:|\alpha|=n} \omega_{1}\left(f_{\alpha}, h\right)\right) k^{n}}{h(n+1) !}\left(\sum_{i=1}^{k} \frac{2^{n+2} \pi^{2}}{3\left(N_{i}-1\right)}\right) \leq \\
\frac{\left(\max _{\alpha:|\alpha|=n} \omega_{1}\left(f_{\alpha}, h\right)\right) k^{n+1}}{h(n+1) !}\left(\frac{2^{n+2} \pi^{2}}{3}\right) \frac{1}{\left(N_{\min }-1\right)}=
\end{gathered}
$$

(setting $\left.h:=\frac{1}{\left(N_{\min }-1\right)}\right)$

$$
\left(\max _{\alpha:|\alpha|=n} \omega_{1}\left(f_{\alpha}, \frac{1}{\left(N_{\min }-1\right)}\right)\right)\left(\frac{2^{n+2} k^{n+1} \pi^{2}}{3(n+1) !}\right),
$$

proving the claim.

We continue with

Theorem 36. Let $[-1,1]^{k}, k \in \mathbb{N}-\{1\}$, with the $l_{1}$-norm $\|\cdot\|$ and $f \in C_{+}\left([-1,1]^{k}\right):|f(t)-f(x)|$ is a convex function in $t \in[-1,1]^{k}$ for a fixed $x \in(-1,1)^{k}$. For a sufficiently large $N_{\min }^{\star}:=\min \left\{N_{1}^{\star}, \ldots, N_{k}^{\star}\right\}$, where $\vec{N}^{\star}:=$ $\left(N_{1}^{\star}, \ldots, N_{k}^{\star}\right) \in \mathbb{N}^{k} ; N_{i}^{\star} \geq 4, i=1, \ldots, k$, the ball in $\mathbb{R}^{k}: B\left(x, \frac{1}{\left(N_{\min }^{\star}-1\right)}\right) \subset[-1,1]^{k}$. Then

$$
\left|L_{\vec{N}}^{(M)}(f)(x)-f(x)\right| \leq\left(\frac{4 \pi^{2} k}{3}\right) \omega_{1}\left(f, \frac{1}{\left(N_{\min }-1\right)}\right),
$$

for all $\vec{N} \in \mathbb{N}^{k}$, where $\vec{N}:=\left(N_{1}, \ldots, N_{k}\right) \in \mathbb{N}^{k}$ and $N_{\min }:=\min \left\{N_{1}, \ldots, N_{k}\right\}$, with $N_{\min } \geq N_{\min }^{\star}$.

It holds that $\lim _{\vec{N} \rightarrow(\infty, \ldots, \infty)} L_{\vec{N}}^{(M)}(f)(x)=f(x)$.

Proof. By (33) we have that

$$
\begin{gathered}
\left|L_{\vec{N}}^{(M)}(f)(x)-f(x)\right| \stackrel{(99)}{\leq} \frac{\omega_{1}(f, h)}{h}\left(\sum_{i=1}^{k} L_{N_{i}}^{(M)}\left(\left|t_{i}-x_{i}\right|\right)\left(x_{i}\right)\right) \\
\stackrel{(94)}{\leq} \frac{\omega_{1}(f, h)}{h}\left(\sum_{i=1}^{k} \frac{4 \pi^{2}}{3\left(N_{i}-1\right)}\right) \leq \frac{\omega_{1}(f, h)}{h}\left(\frac{4 \pi^{2} k}{3}\right) \frac{1}{\left(N_{\min }-1\right)}=
\end{gathered}
$$

(setting $\left.h:=\frac{1}{\left(N_{\min }-1\right)}\right)$

$$
=\omega_{1}\left(f, \frac{1}{\left(N_{\min }-1\right)}\right)\left(\frac{4 \pi^{2} k}{3}\right) \text {, }
$$

proving the claim.

We make 
Remark 37. The Chebyshev knots of first kind $x_{N, k}:=\cos \left(\frac{(2(N-k)+1)}{2(N+1)} \pi\right) \in(-1,1), k \in\{0,1, \ldots, N\}$, $-1<x_{N, 0}<x_{N, 1}<\ldots<x_{N, N}<1$, are the roots of the first kind Chebyshev polynomial $T_{N+1}(x):=$ $\cos ((N+1) \arccos x), x \in[-1,1]$.

Define $(x \in[-1,1])$

$$
h_{N, k}(x):=\left(1-x \cdot x_{N, k}\right)\left(\frac{T_{N+1}(x)}{(N+1)\left(x-x_{N, k}\right)}\right)^{2},
$$

the fundamental interpolation polynomials.

The Max-product interpolation Hermite-Fejér operators on Chebyshev knots of the first kind (see [1, p. 12]) are defined by

$$
H_{2 N+1}^{(M)}(f)(x)=\frac{\bigvee_{k=0}^{N} h_{N, k}(x) f\left(x_{N, k}\right)}{\bigvee_{k=0}^{N} h_{N, k}(x)}, \forall N \in \mathbb{N},
$$

for $f \in C_{+}([-1,1])$, for all $x \in[-1,1]$.

By [1, p. 287], we have

$$
H_{2 N+1}^{(M)}(|\cdot-x|)(x) \leq \frac{2 \pi}{N+1}, \forall x \in[-1,1], \forall N \in \mathbb{N}
$$

And by [4], we get that

$$
H_{2 N+1}^{(M)}\left(|\cdot-x|^{m}\right)(x) \leq \frac{2^{m} \pi}{N+1}, \forall x \in[-1,1], \forall m, N \in \mathbb{N}
$$

Notice $H_{2 N+1}^{(M)}(1)=1$, and $H_{2 N+1}^{(M)}$ maps $C_{+}([-1,1])$ into itself, and it is a positive sublinear operator. Furthermore, it holds that $\bigvee_{k=0}^{N} h_{N, k}(x)>0, \forall x \in[-1,1]$. We also have $h_{N, k}\left(x_{N, k}\right)=1$, and $h_{N, k}\left(x_{N, j}\right)=0$, if $k \neq j$, and $H_{2 N+1}^{(M)}(f)\left(x_{N, j}\right)=f\left(x_{N, j}\right)$, for all $j \in\{0,1, \ldots, N\}$, see [1, p. 282].

We need

Definition 38. Let $f \in C_{+}\left([-1,1]^{k}\right), k \in \mathbb{N}-\{1\}$, and $\vec{N}=\left(N_{1}, \ldots, N_{k}\right) \in \mathbb{N}^{k}$. We define the multivariate Max-product interpolation Hermite-Fejér operators on Chebyshev knots of the first kind, as follows:

$$
\begin{gathered}
H_{2 \vec{N}+1}^{(M)}(f)(x):= \\
\frac{\vee_{i_{1}=0}^{N_{1}} \vee_{i_{2}=0}^{N_{2}} \ldots \vee_{i_{k}=0}^{N_{k}} h_{N_{1}, i_{1}}\left(x_{1}\right) h_{N_{2}, i_{2}}\left(x_{2}\right) \ldots h_{N_{k}, i_{k}}\left(x_{k}\right) f\left(x_{N_{1}, i_{1}}, x_{N_{2}, i_{2}}, \ldots, x_{N_{k}, i_{k}}\right)}{\vee_{i_{1}=0}^{N_{1}} \vee_{i_{2}=0}^{N_{2}} \ldots \vee_{i_{k}=0}^{N_{k}} h_{N_{1}, i_{1}}\left(x_{1}\right) h_{N_{2}, i_{2}}\left(x_{2}\right) \ldots h_{N_{k}, i_{k}}\left(x_{k}\right)},
\end{gathered}
$$

for all $x=\left(x_{1}, \ldots, x_{k}\right) \in[-1,1]^{k}$. Call $N_{\min }:=\min \left\{N_{1}, \ldots, N_{k}\right\}$.

The operators $H_{2 \vec{N}+1}^{(M)}(f)(x)$ are positive sublinear mapping $C_{+}\left([-1,1]^{k}\right)$ into itself, and $H_{2}^{(M)}(1)=1$.

We also have

$$
\begin{gathered}
H_{2 \vec{N}+1}^{(M)}(f)(x):= \\
\frac{\vee_{i_{1}=0}^{N_{1}} \vee_{i_{2}=0}^{N_{2}} \ldots \vee_{i_{k}=0}^{N_{k}} h_{N_{1}, i_{1}}\left(x_{1}\right) h_{N_{2}, i_{2}}\left(x_{2}\right) \ldots h_{N_{k}, i_{k}}\left(x_{k}\right) f\left(x_{N_{1}, i_{1}}, x_{N_{2}, i_{2}}, \ldots, x_{N_{k}, i_{k}}\right)}{\prod_{\lambda=1}^{k}\left(\vee_{i_{\lambda}=0}^{N_{\lambda}} h_{N_{\lambda}, i_{\lambda}}\left(x_{\lambda}\right)\right)},
\end{gathered}
$$

for all $x=\left(x_{1}, \ldots, x_{\lambda}, \ldots, x_{k}\right) \in[-1,1]^{k}$, by the maximum multiplicative principle, see (20). Notice that $H_{2 \vec{N}+1}^{(M)}(f)\left(x_{N_{1}, i_{1}}, \ldots, x_{N_{k}, i_{k}}\right)=f\left(x_{N_{1}, i_{1}}, \ldots, x_{N_{k}, i_{k}}\right)$.

We make

Remark 39. The coordinate Max-product interpolation Hermite-Fejér operators on Chebyshev knots of the first kind, are defined as follows $(\lambda=1, \ldots, k)$ :

$$
H_{2 N_{\lambda}+1}^{(M)}(g)\left(x_{\lambda}\right):=\frac{\vee_{i_{\lambda}=0}^{N_{\lambda}} h_{N_{\lambda}, i_{\lambda}}\left(x_{\lambda}\right) g\left(x_{N_{\lambda}, i_{\lambda}}\right)}{\vee_{i_{\lambda}=0}^{N_{\lambda}} h_{N_{\lambda}, i_{\lambda}}\left(x_{\lambda}\right)},
$$


for all $N_{\lambda} \in \mathbb{N}, x_{\lambda} \in[-1,1]$, and $g \in C_{+}([-1,1])$.

Here we have $\left(\lambda=1, \ldots, k ; x_{\lambda} \in[-1,1]\right)$

$$
h_{N_{\lambda}, i_{\lambda}}\left(x_{\lambda}\right)=\left(1-x_{\lambda} \cdot x_{N_{\lambda}, i_{\lambda}}\right)\left(\frac{T_{N_{\lambda}+1}\left(x_{\lambda}\right)}{\left(N_{\lambda}+1\right)\left(x_{\lambda}-x_{N_{\lambda}, i_{\lambda}}\right)}\right)^{2},
$$

where the Chebyshev knots $x_{N_{\lambda}, i_{\lambda}}=\cos \left(\frac{\left(2\left(N_{\lambda}-i_{\lambda}\right)+1\right)}{2\left(N_{\lambda}+1\right)} \pi\right) \in(-1,1), i_{\lambda} \in\left\{0,1, \ldots, N_{\lambda}\right\},-1<x_{N_{\lambda}, 0}<x_{N_{\lambda}, 1}<$ $\ldots<x_{N_{\lambda}, N_{\lambda}}<1$ are the roots of the first kind Chebyshev polynomial $T_{N_{\lambda}+1}\left(x_{\lambda}\right)=\cos \left(\left(N_{\lambda}+1\right) \arccos x_{\lambda}\right)$, $x_{\lambda} \in[-1,1]$.

In case of $f \in C_{+}\left([-1,1]^{k}\right)$ such that $f(x):=g\left(x_{\lambda}\right)$, for all $x \in[-1,1]^{k}$ and $g \in C_{+}([-1,1])$, we get that

$$
H_{2 \vec{N}+1}^{(M)}(f)(x)=H_{2 N_{\lambda}+1}^{(M)}(g)\left(x_{\lambda}\right),
$$

by the maximum multiplicative principle (20) and simplification of (109).

We present

Theorem 40. Let $x \in(-1,1)^{k}, k \in \mathbb{N}-\{1\}$, be fixed, and let $f \in C^{n}\left([-1,1]^{k}, \mathbb{R}_{+}\right), n \in \mathbb{N}$. We assume that $f_{\alpha}(x)=0$, for all $\alpha:|\alpha|=1, \ldots, n$. Here $\left|f_{\alpha}\right|$ is assumed to be convex over $[-1,1]^{k}$, all $\alpha:|\alpha|=n$. The set $[-1,1]^{k}, k \in \mathbb{N}-\{1\}$ is endowed with the $l_{1}$-norm and $\omega_{1}\left(f_{\alpha}, \cdot\right)$, all $\alpha:|\alpha|=n$ is with respect to $l_{1}$-norm. For a sufficiently large $N_{\min }^{\star}:=\min \left\{N_{1}^{\star}, \ldots, N_{k}^{\star}\right\}$, where $\vec{N}^{\star}:=\left(N_{1}^{\star}, \ldots, N_{k}^{\star}\right) \in \mathbb{N}^{k}$, the ball in $\mathbb{R}^{k}: B\left(x, \frac{1}{\left(N_{\min }^{*}+1\right)}\right) \subset$ $[-1,1]^{k}$. Then

$$
\left|H_{2 \vec{N}+1}^{(M)}(f)(x)-f(x)\right| \leq\left(\frac{(2 k)^{n+1} \pi}{(n+1) !}\right)\left(\max _{\alpha:|\alpha|=n} \omega_{1}\left(f_{\alpha}, \frac{1}{\left(N_{\min }+1\right)}\right)\right),
$$

for all $\vec{N} \in \mathbb{N}^{k}$, where $\vec{N}:=\left(N_{1}, \ldots, N_{k}\right) \in \mathbb{N}^{k}$ and $N_{\min }:=\min \left\{N_{1}, \ldots, N_{k}\right\}$, with $N_{\min } \geq N_{\min }^{\star}$.

It holds that $\lim _{\vec{N} \rightarrow(\infty, \ldots, \infty)} H_{2 \vec{N}+1}^{(M)}(f)(x)=f(x)$.

Proof. By (19) we get:

$$
\begin{gathered}
\left|H_{2 \vec{N}+1}^{(M)}(f)(x)-f(x)\right|_{\leq}^{(112)} \frac{\left(\max _{\alpha:|\alpha|=n} \omega_{1}\left(f_{\alpha}, h\right)\right) k^{n}}{h(n+1) !} . \\
\left(\sum_{i=1}^{k} H_{2 N_{i}+1}^{(M)}\left(\left|t_{i}-x_{i}\right|^{n+1}\right)\left(x_{i}\right)\right) \stackrel{(107)}{\leq} \\
\frac{\left(\max _{\alpha:|\alpha|=n} \omega_{1}\left(f_{\alpha}, h\right)\right) k^{n}}{h(n+1) !}\left(\sum_{i=1}^{k} \frac{2^{n+1} \pi}{\left(N_{i}+1\right)}\right) \leq \\
\frac{\left(\max _{\alpha:|\alpha|=n} \omega_{1}\left(f_{\alpha}, h\right)\right) k^{n+1}}{h(n+1) !}\left(2^{n+1} \pi\right) \frac{1}{\left(N_{\min }+1\right)}=
\end{gathered}
$$

(setting $\left.h:=\frac{1}{\left(N_{\min }+1\right)}\right)$

$$
\left(\max _{\alpha:|\alpha|=n} \omega_{1}\left(f_{\alpha}, \frac{1}{\left(N_{\min }+1\right)}\right)\right)\left(\frac{2^{n+1} k^{n+1} \pi}{(n+1) !}\right)
$$

proving the claim.

We continue with 
Theorem 41. Let $[-1,1]^{k}, k \in \mathbb{N}-\{1\}$, with the $l_{1}$-norm $\|\cdot\|$ and $f \in C_{+}\left([-1,1]^{k}\right):|f(t)-f(x)|$ is a convex function in $t \in[-1,1]^{k}$ for a fixed $x \in(-1,1)^{k}$. For a sufficiently large $N_{\min }^{\star}:=\min \left\{N_{1}^{\star}, \ldots, N_{k}^{\star}\right\}$, where $\vec{N}^{\star}:=$ $\left(N_{1}^{\star}, \ldots, N_{k}^{\star}\right) \in \mathbb{N}^{k}$, the ball in $\mathbb{R}^{k}: B\left(x, \frac{1}{\left(N_{\min }^{\star}+1\right)}\right) \subset[-1,1]^{k}$. Then

$$
\left|H_{2 \vec{N}+1}^{(M)}(f)(x)-f(x)\right| \leq 2 k \pi \omega_{1}\left(f, \frac{1}{\left(N_{\min }+1\right)}\right),
$$

for all $\vec{N} \in \mathbb{N}^{k}$, where $\vec{N}:=\left(N_{1}, \ldots, N_{k}\right) \in \mathbb{N}^{k}$ and $N_{\min }:=\min \left\{N_{1}, \ldots, N_{k}\right\}$, with $N_{\min } \geq N_{\min }^{\star}$.

It holds that $\lim _{\vec{N} \rightarrow(\infty, \ldots, \infty)} H_{2 \vec{N}+1}^{(M)}(f)(x)=f(x)$.

Proof. By (33) we have that

$$
\begin{gathered}
\left|H_{2 \vec{N}+1}^{(M)}(f)(x)-f(x)\right| \stackrel{(112)}{\leq} \frac{\omega_{1}(f, h)}{h}\left(\sum_{i=1}^{k} H_{2 N_{i}+1}^{(M)}\left(\left|t_{i}-x_{i}\right|\right)\left(x_{i}\right)\right) \\
\quad \stackrel{\leq}{\leq} \frac{\omega_{1}(f, h)}{h}\left(\sum_{i=1}^{k} \frac{2 \pi}{\left(N_{i}+1\right)}\right) \leq \frac{\omega_{1}(f, h)}{h}(2 \pi k) \frac{1}{\left(N_{\min }+1\right)}=
\end{gathered}
$$

(setting $\left.h:=\frac{1}{\left(N_{\min }+1\right)}\right)$

$$
=\omega_{1}\left(f, \frac{1}{\left(N_{\min }+1\right)}\right)(2 k \pi),
$$

proving the claim.

We give

Theorem 42. Let $Q$ with the $l_{1}$-norm $\|\cdot\|$, be a compact and convex subset of $\mathbb{R}^{k}, k \in \mathbb{N}-\{1\}$, and $f \in C_{+}(Q)$ : $|f(t)-f(x)|$ is a convex function in $t \in Q$ for a fixed $x \in Q^{\circ}$. The first modulus of continuity $\omega_{1}(f, \cdot)$ is with respect to $l_{1}$-norm. Let $\left\{L_{N}\right\}_{N \in \mathbb{N}}$ be positive sublinear operators from $C_{+}(Q)$ into itself: $L_{N}(1)=1$, for all $N \in \mathbb{N}$. We assume that $L_{N}(\|t-x\|)(x)>0$ and the ball in $\mathbb{R}^{k}: B\left(x, L_{N}(\|t-x\|)(x)\right) \subset Q$, for all $N \in \mathbb{N}$. Then

$$
\left|L_{N}(f)(x)-f(x)\right| \leq \omega_{1}\left(f, L_{N}(\|t-x\|)(x)\right), \forall N \in \mathbb{N} \text {. }
$$

If $L_{N}(\|t-x\|)(x) \rightarrow 0$, then $L_{N}(f)(x) \rightarrow f(x)$, as $N \rightarrow+\infty$.

Proof. By (33).

We need

Theorem 43. All as in Theorem $2, f \in C^{n}\left(Q, \mathbb{R}_{+}\right)$. Let $\left\{L_{N}\right\}_{N \in \mathbb{N}}$ be positive sublinear operators mapping $C_{+}(Q)$ into itself, such that $L_{N}(1)=1$, for all $N \in \mathbb{N}$. Then

$$
\left|L_{N}(f)(x)-f(x)\right| \leq \frac{\left(\max _{\alpha:|\alpha|=n} \omega_{1}\left(f_{\alpha}, h\right)\right)}{h(n+1) !}\left(L_{N}\left(\|t-x\|^{n+1}\right)\right)(x),
$$

for all $N \in \mathbb{N}$.

Proof. By (12), see Definition 3, and by (18).

We finally present

Theorem 44. Let $Q$ with the $l_{1}$-norm $\|\cdot\|$, be a compact and convex subset of $\mathbb{R}^{k}, k \geq 1$, let $x \in Q^{o}$ be fixed. Let $f \in C_{+}^{n}\left(Q, \mathbb{R}_{+}\right), n \in \mathbb{N}$, and $f_{\alpha}$ with $\alpha:|\alpha|=n$, has a first modulus of continuity $\omega_{1}\left(f_{\alpha}, \cdot\right)$ relative to $Q$ with respect to $l_{1}$-norm. Each $\left|f_{\alpha}\right|$ is a convex function over $Q$, all $\alpha:|\alpha|=n$. Assume further that $f_{\alpha}(x)=0$, 
all $\alpha:|\alpha|=1, \ldots, n$. Let $\left\{L_{N}\right\}_{N \in \mathbb{N}}$ be positive sublinear operators from $C_{+}(Q)$ into $C_{+}(Q): L_{N}(1)=1$, for all $N \in \mathbb{N}$. We further assume that $L_{N}\left(\|t-x\|^{n+1}\right)(x)>0$ and the ball in $\mathbb{R}^{k}: B\left(x, L_{N}\left(\|t-x\|^{n+1}\right)(x)\right) \subset Q$, for all $N \in \mathbb{N}$. Then

$$
\left|L_{N}(f)(x)-f(x)\right| \leq \frac{\left(\max _{\alpha:|\alpha|=n} \omega_{1}\left(f_{\alpha},\left(L_{N}\left(\|t-x\|^{n+1}\right)\right)(x)\right)\right)}{(n+1) !}, \forall N \in \mathbb{N} .
$$

It holds, as $L_{N}\left(\|t-x\|^{n+1}\right)(x) \rightarrow 0$, then $L_{N}(f)(x) \rightarrow f(x)$, when $N \rightarrow+\infty$.

Proof. By (118).

\section{References}

[1] Bede B., Coroianu L., Gal S., Approximation by Max-Product type Operators, Springer, Heidelberg, New York, 2016

[2] Anastassiou G., Moments in probability and approximation theory, Pitman Research Notes in Mathematics Series, Longman Group UK, New York, NY, 1993

[3] Anastassiou G., Approximation by Sublinear Operators, submitted, 2017

[4] Anastassiou G., Approximation by Max-Product Operators, submitted, 2017 